\title{
Fuzzi: A Three-Level Logic for Differential Privacy
}

HENGCHU ZHANG, University of Pennsylvania, USA

EDO ROTH, University of Pennsylvania, USA

ANDREAS HAEBERLEN, University of Pennsylvania, USA

BENJAMIN C. PIERCE, University of Pennsylvania, USA

AARON ROTH, University of Pennsylvania, USA

Curators of sensitive datasets sometimes need to know whether queries against the data are differentially private.Two sorts of logics have been proposed for checking this property: (1) type systems and other static analyses, which fully automate straightforward reasoning with concepts like "program sensitivity" and "privacy loss," and (2) full-blown program logics such as apRHL (an approximate, probabilistic, relational Hoare logic),which support more flexible reasoning about subtle privacy-preserving algorithmic techniques but offer only minimal automation.

We propose a three-level logic for differential privacy in an imperative setting and present a prototype implementation called Fuzzi. Fuzzi's lowest level is a general-purpose logic; its middle level is apRHL; and its top level is a novel sensitivity logic adapted from the linear-logic-inspired type system of Fuzz, a differentially private functional language.The key novelty is a high degree of integration between the sensitivity logic and the two lower-level logics: the judgments and proofs of the sensitivity logic can be easily translated into apRHL; conversely, privacy properties of key algorithmic building blocks can be proved manually in apRHL and the base logic, then packaged up as typing rules that can be applied by a checker for the sensitivity logic to automatically construct privacy proofs for composite programs of arbitrary size.

We demonstrate Fuzzi's utility by implementing four different private machine-learning algorithms and showing that Fuzzi's checker is able to derive tight sensitivity bounds.

CCS Concepts: • Theory of computation $\rightarrow$ Programming logic; Program specifications.

Additional Key Words and Phrases: Differential privacy, typechecking, static analysis, apRHL, Fuzz, Fuzzi

ACM Reference Format:

Hengchu Zhang, Edo Roth, Andreas Haeberlen, Benjamin C. Pierce, and Aaron Roth. 2019. Fuzzi: A ThreeLevel Logic for Differential Privacy. Proc. ACM Program. Lang. 3, ICFP, Article 93 (August 2019), 28 pages. https://doi.org/10.1145/3341697

\section{INTRODUCTION}

Differential privacy [Dwork et al. 2006] has become the gold standard for privacy-preserving statistical analysis in the academic community, and it is being adopted by a growing number of industry and government organizations, including Apple [Apple 2017], Google [Erlingsson et al. 2014], Microsoft [Microsoft 2017] and the US Census Bureau [N. Dajani et al. 2017]. Differential privacy makes minimal assumptions about an adversary's knowledge, allowing analysts to quantitatively estimate privacy loss. However, the reasoning needed to correctly achieve differential privacy can

Authors' addresses: Hengchu Zhang, University of Pennsylvania, USA, hengchu@seas.upenn.edu; Edo Roth, University of Pennsylvania, USA, edoroth@seas.upenn.edu; Andreas Haeberlen, University of Pennsylvania, USA, ahae@cis.upenn.edu; Benjamin C. Pierce, University of Pennsylvania, USA, bcpierce@cis.upenn.edu; Aaron Roth, University of Pennsylvania, USA, aaroth@cis.upenn.edu.

This work is licensed under a Creative Commons Attribution 4.0 International License.

(C) 2019 Copyright held by the owner/author(s).

2475-1421/2019/8-ART93

https://doi.org/10.1145/3341697

Proc. ACM Program. Lang., Vol. 3, No. ICFP, Article 93. Publication date: August 2019. 
be rather subtle, as multiple errors in published algorithms attest [Chen and Machanavajjhala 2015; Lyu et al. 2016].

Barthe et al. [2016] developed the first program logic for formalizing proofs of differential privacy for imperative programs, called apRHL (Approximate, Probabilistic, Relational Hoare Logic). The abstractions provided by apRHL are expressive enough to capture the essence of many complex differentially private algorithms, allowing experts to prove differential privacy for small, tricky code sequences at a fairly high level of abstraction. However, proving differential privacy in apRHL for large programs can be a rather tedious endeavor. Fortunately, in many proofs for larger private data analysis programs (either in apRHL or on paper), the expert knowledge of differential privacy is concentrated in the analysis of small differentially private subroutines, while the rest of the proof basically just propagates "sensitivity" information and aggregate privacy costs between subroutines. This suggests that one could considerably increase the range of possible use cases, especially for analysts who are not privacy experts, by combining a small but extensible set of building blocks (and the corresponding manual proofs) with a largely automated analysis that mechanically completes the proof for a given program.

To enable this approach, we build a new layer of abstraction over apRHL to automate the mechanical parts of this process. This layer tracks sensitivities for program variables and privacy costs of commands using Hoare-triple-style proof rules. This information about sensitivity and privacy cost has a direct translation to lower-level apRHL assertions. This allows information in the higher-level logic to seamlessly interact with expert proofs of differential privacy that have been carried out using the two lower layers. Since the top layer is entirely automated, we will often refer to it as a type system (and to its proof rules as typing rules).

We use the term mechanisms to refer to building blocks of differentially private programs. Many differentially private mechanisms can be viewed as parameterized program templates, where the differential privacy properties depend on properties of the instantiation parameters, which can themselves be program expressions or commands. In order to integrate expert reasoning about such mechanisms, we develop a framework for expressing program templates and the corresponding parameterized proofs of differential privacy. This allows experts to extend the sensitivity type system with a specialized typing rule for each template, allowing non-expert programmers to write application programs that combine these templates in straightforward ways. This framework uses apRHL directly to give structured proofs of privacy property, while using the general-purpose base logic to establish lower-level semantic properties that go beyond the capabilities of apRHL.

We instantiate these ideas in the design and implementation of Fuzzi, a small imperative language for differentially private queries with automatic and extensible typechecking. Following a brief review of technical background on differential privacy (Section 2) and a high-level overview of Fuzzi's design (Section 3), we offer the following contributions:

(1) We propose a high-level sensitivity logic for tracking differential privacy (Section 4). This logic is expressive enough to capture detailed sensitivity properties for a simple imperative core language; its soundness is established via a straightforward embedding into apRHL.

(2) We show how to connect manual proofs for privacy properties of algorithmic building blocks to the sensitivity logic and develop proofs for several mechanisms that transform private datasets, plus a mechanism that aggregates privacy costs better than straightforward composition (Section 5).

(3) Using a prototype implementation of Fuzzi (Section 6), we implement private machine learning algorithms from four different classes of learning methods (discriminative models, ensemble models, generative models and instance-based learning) and show that Fuzzi's checker is able to derive tight sensitivity bounds (Section 7). 
Section 8 discusses limitations of the current design. Sections 9 and 10 survey related and future work.

\section{BACKGROUND}

\subsection{Differential Privacy}

Differential privacy is an indistinguishability property of randomized programs on neighboring input datasets. Informally, a function is differentially private if removing or adding a single row in the input results in at most a small change in the output distribution.

Definition 1 (Neighboring Dataset). Two datasets are neighbors if one can be transformed into the other by adding or removing a single row of data.

Let $D$ and $D^{\prime}$ be two neighboring datasets, and let $f$ be a randomized program. The output of $f$ is a sample from some distribution parameterized by the input datasets. We write $f(D)$ and $f\left(D^{\prime}\right)$ for these two distributions.

Definition $2((\epsilon, \delta)$-Differential Privacy [Dwork et Al. 2006]). The program $f$ is $(\epsilon, \delta)$ differentially private if, for any set of possible outputs $E$, the probability of observing $E$ satisfies the relation $\mathbb{P}_{x \sim f(D)}[x \in E] \leq e^{\epsilon} \mathbb{P}_{x \sim f\left(D^{\prime}\right)}[x \in E]+\delta$.

The parameters $\epsilon$ and $\delta$ quantify different aspects of the privacy cost of a differentially private computation. Informally, the value of $\epsilon$ measures the ability of an observer to distinguish whether $f$ was run with $D$ or $D^{\prime}$ after observing $E$ in the "common case", while $\delta$ serves as an upper bound on the probability that $f$ fails to provide the privacy guarantee implied by $\epsilon$. The parameter $\epsilon$ is typically taken to be a small constant (say, 1), whereas $\delta$ must be set so that $\delta \ll 1 / n$, where $n$ is the number of dataset rows, in order for the privacy guarantees to be non-trivial (otherwise an algorithm which outputs a dataset row uniformly at random satisfies $(0, \delta)$-differential privacy).

\subsection{Sensitivity}

The notion of sensitivity is crucial to differential privacy. Many differentially private mechanisms release data by adding noise proportional to the sensitivity of a private value. In Fuzzi, the term "sensitivity" specifically refers to an upper bound on the distance between the values held by some variable between any two runs.

Distance may be calculated differently for values of different types. For primitive values with type int and real, distance is the magnitude of the two values' difference. However, for arrays, there are two important distance definitions for differential privacy: database distance and L1 distance. The database distance measures the number of rows that need to be added or removed in order to make two datasets indistinguishable up to permutation; while the L1 distance measures the sum of element-wise distance between vectors. To avoid confusion in later discussions, we refer to arrays for which distance is intended to be measured as database distance as bags and arrays with L1 distance as vectors. When we come to defining the type system, we will write $\{\tau\}$ for the type of bags holding values of type $\tau$ and $[\tau]$ for vectors of $\tau$ (Figure 1). As an example, the two arrays $[1,2,5]$ and $[1,3,4]$ have vector distance 2 , but they have bag distance 4 , since we need to remove elements 2 and 5 and add elements 3 and 4 to the first bag in order to make it a permutation of the second one. ${ }^{1}$

\footnotetext{
${ }^{1}$ Database distance can actually be viewed as just L1 distance on a different representation of datasets. The differential privacy literature sometimes uses the "histogram representation" for datasets. For a universe of possible elements $U$, the histogram representation maps each $x \in U$ to a count of how many times $x$ appears, and the L1 distance of this representation corresponds to the database distance. However, in order to keep Fuzzi's semantics minimal as a core language we choose to represent datasets as arrays, rather than maps from records to counts.
} 


$$
\begin{aligned}
& \sigma:=\text { int | real | bool } \\
& \tau:=\sigma|[\tau]|\{\tau\} \\
& \text { op }:=+|-| \cdot|/| \& \&|||| \\
& <|\leq|>|\geq| \neg \\
& e, i:=x \mid \text { lit } \mid \text { eope }|e[i]| \text { e.length }
\end{aligned}
$$

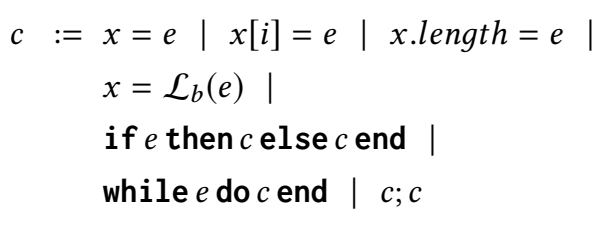

Fig. 1. Core Language Syntax

Formally, we write $d_{\tau}$ for the distance function at type $\tau$, with type $\tau \times \tau \rightarrow \mathbb{R}^{+} \cup\{\infty\}$-i.e., it maps two values of type $\tau$ to a non-negative real number or infinity.

Definition 3 (Vector Distance). If $a_{1}$ and $a_{2}$ are two vectors of the same length and their elements have type $\tau$, then the vector distance is defined as $d_{[\tau]}\left(a_{1}, a_{2}\right)=\sum_{i=0}^{L-1} d_{\tau}\left(a_{1}[i], a_{2}[i]\right)$ where $L$ is the length of both vectors. Vectors of different lengths are assigned the distance $\infty$.

Definition 4 (BAg Distance). Let $a_{1}$ and $a_{2}$ be two bags; their distance is defined as $d_{\{\tau\}}\left(a_{1}, a_{2}\right)=$ $\left|a_{1} \backslash a_{2}\right|+\left|a_{2} \backslash a_{1}\right|$.

The backslash operator is multiset difference. Note that bag distance (unlike vector distance) is meaningful for bags of different sizes. This is the same database distance introduced earlier.

\subsection{Laplace Mechanism}

The Laplace mechanism is an essential tool for releasing private data with bounded sensitivities [Dwork et al. 2006; Dwork and Roth 2014]. Fuzzi provides access to the Laplace mechanism through the sampling assignment command $x=\mathcal{L}_{b}(e)$, which adds noise to the value of $e$ and assigns that value to the variable $x$, with the constant literal $b$ determining the scale of the noise. Adding noise scaled with $b$ to a value with sensitivity $s$ incurs a privacy cost of $(s / b, 0)$. Fuzzi's type system will statically keep track of each usage of the Laplace mechanism and report an upper bound of the total privacy cost as part of a program's type.

\section{OVERVIEW}

\subsection{Core Language}

The core of Fuzzi is a simple imperative programming language with while loops, conditionals, and assignments (Figure 1). It has just a few built-in data types: reals, integers, booleans, and arrays (whose elements can be reals, integers, booleans, or nested arrays). Programs can modify the length of arrays through assignments of the form $x$.length $=e$. When the value of $e$ is less than the current length of $x$, the array is truncated; and when the value of $e$ is greater than the length of $x$, the array is padded with default values that are of the same data type as elements in $x$. If $e$ evaluates to a negative number, the length assignment diverges.

One slightly unusual feature of Fuzzi is that all assignments are copying assignments, including assignments to array variables. For example, if $x$ holds an array value, then the assignment $y=x$ sets $y$ to a copy of $x$, instead of making both $x$ and $y$ point to the same underlying array. We make this choice to avoid reasoning about sharing, which we consider as out of scope for this work.

The special command $x=\mathcal{L}_{b}(e)$ performs probabilistic assignment to $x$ by sampling from a Laplace distribution centered at the value of $e$, with width equal to the value of $b$ (which must be a real-valued literal). 


\subsection{Type System}

From now on, we refer to the sensitivity logic as a type system to emphasize that it is a specialized and automated layer that tracks sensitivity and privacy cost as types. The main data structure manipulated by Fuzzi's type system is typing contexts-maps from program variables to sensitivities, represented as non-negative reals extended with infinity.

$$
\phi:=r \in \mathbb{R}^{\geq 0} \cup\{\infty\} \quad \tau:=\sigma|[\tau]|\{\tau\} \quad \Gamma:=\varnothing \mid x:{ }_{\phi} \tau, \Gamma
$$

A typing context $\Gamma$ should be interpreted as a relation between two different run-time storesintuitively, the stores arising from two "neighboring" executions starting with neighboring initial states. For each variable $x$ with sensitivity $\Gamma(x)$, the values in the two stores must be no more than $\Gamma(x)$ apart.

Typing judgements for commands have the form $\{\Gamma\} c\left\{\Gamma^{\prime},(\epsilon, \delta)\right\}$, meaning that, if the distance of the values in two run-time stores are described by the initial typing context $\Gamma$, then executing $c$ will either both diverge or else both terminate with two final stores described by $\Gamma^{\prime}$, along the way incurring a privacy cost of $(\epsilon, \delta)$.

For example, the typing rule for commands of the form $x=e$ computes the sensitivity of $e$ using sensitivities of its free variables, and maps $x$ to this sensitivity of $e$ in the output context.

$$
\frac{\Gamma \vdash e \epsilon_{s} \tau}{\{\Gamma\} x=e\{\Gamma[x \mapsto s],(0,0)\}}
$$

The typechecker also computes the privacy cost $(\epsilon, \delta)$ incurred by the analyzed command. In the case of assignment, no privacy cost is incurred, so the output from the typechecker after processing $x=e$ is the updated typing context $\Gamma[x \mapsto s]$ and the pair of privacy costs $(0,0)$, where $s$ is the derived sensitivity of $e$ under $\Gamma$.

A more interesting typing rule is the one for sequence commands of the form $c_{1} ; c_{2}$. This rule chains together the typing judgements for each of the commands, using the output context $\Gamma_{i}$ from analyzing $c_{i}$ as the input context for processing the next command $c_{i+1}$. The privacy cost incurred by the whole program is the sum of privacy costs $\left(\epsilon_{i}, \delta_{i}\right)$ incurred by each $c_{i}$, following the "simple composition theorem" for differential privacy [Dwork et al. 2006].

$$
\begin{aligned}
& \text { SEQUENCE } \\
& \frac{\left\{\Gamma_{1}\right\} c_{1}\left\{\Gamma_{2},\left(\epsilon_{1}, \delta_{1}\right)\right\} \quad\left\{\Gamma_{2}\right\} c_{2}\left\{\Gamma_{3},\left(\epsilon_{2}, \delta_{2}\right)\right\}}{\left\{\Gamma_{1}\right\} c_{1} ; c_{2}\left\{\Gamma_{3},\left(\epsilon_{1}+\epsilon_{2}, \delta_{1}+\delta_{2}\right)\right\}}
\end{aligned}
$$

There are also core typing rules for simple loops and conditionals that do not branch on sensitive data; we will see these in Section 4.1.

\subsection{Typing Differentially Private Mechanisms}

The privacy properties of interesting differentially private mechanisms are generally too subtle to be tracked by the core type system. In Fuzzi, such mechanisms can be defined as extensions and equipped with specialized typing rules whose soundness is proved manually. Such proofs typically involve reasoning about relational properties for distributions, as well as aggregating privacy costs. The program logic apRHL is tailored to tackle both problems, making it a good choice for rigorous manual proofs of differential privacy.

An apRHL judgement has the form $\vdash c_{1} \sim_{(\epsilon, \delta)} c_{2}: \Phi \Rightarrow \Psi$, where $c_{1}$ and $c_{2}$ are two commands to be related, $\Phi$ and $\Psi$ are relational assertions that state pre- and post-conditions relating the program states before and after executing $c_{1}$ and $c_{2}$. A sound apRHL judgement can be roughly interpreted as: if (1) some pair of program states satisfy the pre-condition $\Phi$, and (2) executing $c_{1}$ in the first state terminates iff executing $c_{2}$ in the second state does, then the pair of states 
after executing $c_{1}$ and $c_{2}$ will satisfy the post-condition $\Psi$, incurring privacy cost $(\epsilon, \delta)$. To express differential privacy as a post-condition, we can simply state out $\langle 1\rangle=$ out $\langle 2\rangle$ for the output of the programs.

Given a typing context $\Gamma$, we can interpret $\Gamma$ as a relation on program states. Writing $\langle 1\rangle$ and $\langle 2\rangle$ after variables to refer to their values in the first or second execution, we translate $x:_{\sigma} \tau \in \Gamma$ to the assertion $d_{\tau}(x\langle 1\rangle, x\langle 2\rangle) \leq \sigma$; the conjunction of all these pointwise distance assertions forms an apRHL assertion that corresponds to $\Gamma$.

Conversely, to connect manual proofs in apRHL with the type system, we phrase their premises and conclusions as typing judgements. Indeed, we use apRHL not only for extensions but also for the soundness proofs of the core typing rules. As a result, the privacy proofs implicitly constructed by the typechecker are combinations of apRHL proof objects, some of them generated by the typechecker, others written manually by experts.

\subsection{Example}

To give a first taste of Fuzzi's differential privacy typechecking process, we present a simple program that computes a private approximation for the average income of a group through private estimations of the group's size and sum. First, we estimate the group's size with the Laplace mechanism.

size $=\mathcal{L}_{1.0}$ (group.length);

Assuming that group is a dataset with sensitivity 1, Fuzzi's typechecker deduces its size is 1-sensitive. Applying the Laplace mechanism then incurs a (1.0,0)-privacy cost.

Next, we sum the group's incomes using the mechanism bsum, pronounced "bag sum", which clips each income value so that its magnitude is at most a given constant (here 1000).

bsum (group, sum, i, temp, 1000);

This clipping step ensures the sum does not vary too much on neighboring datasets. Without clipping, a single outlier could sway the sum substantially, revealing the outlier's existence, and violating differential privacy. The parameters sum, $i$, and temp specify the names of variables that bsum can use for internal purposes. It is the programmer's responsibility to make sure they do not clash with variables used elsewhere in the program. (It should not be hard to fix this infelicity by making extensions themselves deal with fresh variable generation, but doing so will introduce a few additional technicalities so we leave it for future work.)

The command bsum (...) refers to an extension that expands to a sequence of plain core-language commands implementing summing up a bag of numbers with clipping:

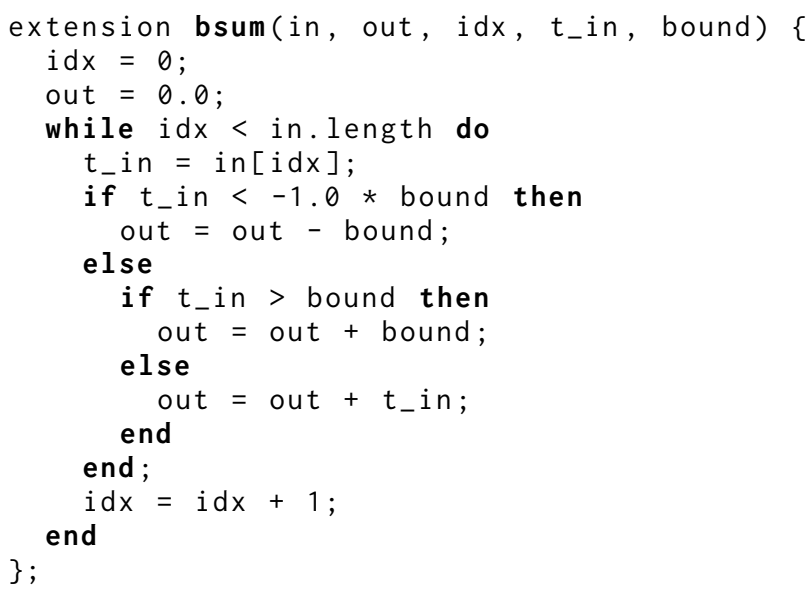


This specifies the name of the extension, the names of its parameters (which range over Fuzzi expressions and commands), and how it expands to core Fuzzi commands. During typechecking, extension applications are replaced by their expansions, with extension variables substituted by the snippets of Fuzzi syntax provided as parameters. )

The typing rule for bsum is:

$$
\begin{array}{cc} 
& \text { literal bound } \quad \text { bound } \geq 0 \\
\phi=\Gamma(\text { in }) & \Gamma_{\text {out }}=\Gamma[\text { out } \mapsto \phi \cdot \text { bound }]\left[i, t_{\text {in }} \mapsto \infty\right] \\
\hline
\end{array}
$$

$\{\Gamma\} \operatorname{bsum}\left(\right.$ in, out $, i, t_{\text {in }}$, bound $)\left\{\Gamma_{\text {out }},(0,0)\right\}$

It requires the last parameter bound to be a non-negative literal value-non-negative because bound specifies the clipping magnitude, and literal because the sensitivity of the output variable depends on bound. The inference rule updates the sensitivity of the output sum variable to the product of bound and the sensitivity of $\Gamma(\mathrm{in})$. Intuitively, since up to $\phi$ elements may be added or removed from in, and each can contribute up a value with magnitude up to bound toward the sum, the sum value itself will vary by at most $\phi \cdot$ bound. This intuition can be made rigorous, as we show in Appendix D. 4 of the extended version.

The Haskell implementation of the Fuzzi typechecker is likewise extended with a piece of code implementing the typing rule as a function that transforms an input typing context to an output typing context and privacy costs.

Continuing the example, we next compute differentially private estimates of the clipped sums and calculate the group's average income using the size and sum estimates:

noised_sum $=\mathcal{L}_{1000.0}($ sum $)$;

$\operatorname{avg}=$ noised_sum / size;

The sum variable is 1000 -sensitive, so releasing noised_sum incurs another $(1.0,0)$-privacy cost. The typechecker reports an aggregate privacy cost of $(2.0,0)$.

\section{SENSITIVITY TYPE SYSTEM}

\subsection{Notation and Definitions}

Throughout the paper, we will use the operator $\llbracket \cdot \|$ to denote the semantic function for commands and expressions in Fuzzi. We use the notation $\bigcirc S$ to denote sub-distributions over values in $S$. We will use the letter $M, N$ to stand for program states, which are finite maps from variable names to the values they hold, and use the letter $\mathbb{M}$ to stand for the set of all program states.

The semantics of a Fuzzi program $c$ is a function from program states to sub-distributions over program states $\llbracket c \rrbracket: \mathbb{M} \rightarrow \bigcirc \mathbb{M}$. Each type in Fuzzi is associated with a set of values: int with the set $\mathbb{Z}$, real with the set $\mathbb{R}$, and $[\tau]$ and $\{\tau\}$ with the set of finite sequences of values associated with $\tau$. The meaning of a Fuzzi expression $e$ with type $\tau$ is a partial function from program states to associated values of that type $\llbracket e \rrbracket: \mathbb{M} \rightarrow \tau$. Partiality of expressions stems from invalid operations such as arithmetic between incompatible values, and out-of-bound indexing. The complete definition of Fuzzi semantics can be found in Appendix A of the extended version. Recall from Section 3.1 that Fuzzi assignments are copy-assignments for all values, including vectors and bags.

Fuzzi's semantics directly follows from the work of Barthe et al. [2016]. It is worth noting that the original apRHL developed by Barthe et al. [2016] only reasons with discretized Laplace distributions, and Fuzzi shares this restriction in its semantic model. A later model based on category theory enhances apRHL's proof rules for continuous distributions [Sato 2016]. However, the underlying proof method of this model is not compatible with Fuzzi's development, and only recently have 


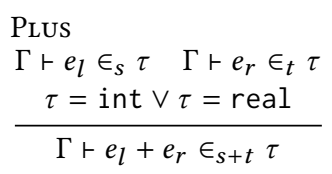

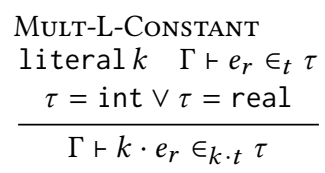

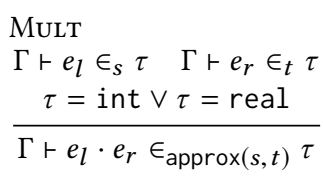

Fig. 2. Arithmetic Expression Typing Rules

new abstractions been proposed to generalize the original apRHL proof methods to continuous distributions [Sato et al. 2019].

Fuzzi's typing context $\Gamma$ tracks both the data type and the sensitivity of variables. Typechecking involves checking data types as well as computing sensitivities. We refer to data typechecking as shape checking and sensitivity computations as sensitivity checking. We will elide details of shape checking since it is the standard typechecking that rules out operations between values of incompatible types. To emphasize sensitivity checking in Fuzzi, and to reduce clutter in syntax, we will write $\Gamma(x)$ for the sensitivity of the variable $x$ under the typing context $\Gamma$, and we write $\Gamma[x \mapsto s]$ for a typing context which updates variable $x$ 's sensitivity to $s$, but does not alter its data type. We overload this syntax when we update a set of variables $x s$ to the same sensitivity $\Gamma[x s \mapsto s]$. We also overload the notation $\Gamma(e)$ to denote the derived sensitivity of expression $e$ under typing context $\Gamma$. When we need to refer to the data type of expression $e$, we will use the full typing judgment of an expression $\Gamma \vdash e \epsilon_{\phi} \tau$, which we pronounce "expression $e$ has sensitivity $\phi$ and type $\tau$ under context $\Gamma$."

We use the notation shape $(\Gamma)$ to extract the shape checking context from a typing context $\Gamma$, dropping all sensitivity annotations.

\subsection{Typing Expressions}

In order to compute sensitivity updates throughout sequences of commands, the type system needs to first compute sensitivities for expressions used within each command. We discuss the typing rules for addition and multiplication here as examples. Intuitively, if the values of two expressions $e_{l}$ and $e_{r}$ can each vary by 1 , then their sum can vary by at most 2 (the sum of their individual sensitivities) by the triangle inequality; and if the value of $e$ can vary by at most 1, then multiplying $e$ by a literal constant $k$ results in a value that can vary by at most $k$. The rules Plus, and Mult-L-Constant in Figure 2 capture these cases.

There are also expressions for which we cannot give precise sensitivity bounds. For instance, if one of the operands for a multiplication between $e_{l}$ and $e_{r}$ is sensitive, then, without knowing the exact value of the other operand, we cannot a priori know how much the value of entire product can change. This case is captured by the MulT rule, where the function approx is defined by the equations

$$
\begin{array}{ll}
\text { approx }(0,0) & =0 \\
\text { approx }\left(s_{1}, s_{2}\right) & \text { if } s_{1}+s_{2}>0=
\end{array}
$$

which conservatively take the sensitivity to be $\infty$ if at least one side of the expression is sensitive.

Fuzzi provides bag and vector index operations, and Fuzzi's typechecker supports sensitivity checking for lookup expressions on bags and vectors. These typing rules use the definition of bag and vector distances to establish sound upper bounds of sensitivities on lookup expressions.

$$
\begin{aligned}
& \text { Vector-Index } \\
& \Gamma \vdash e \epsilon_{\phi}[\tau] \quad \Gamma \vdash i \epsilon_{0} \text { int } \\
& \phi<\infty \\
& \hline \Gamma \vdash e[i] \epsilon_{\phi} \tau
\end{aligned}
$$

$$
\begin{aligned}
& \text { BAG-INDEX } \\
& \Gamma \vdash e \in_{0}\{\tau\} \quad \Gamma \vdash i \epsilon_{0} \text { int } \\
& \Gamma \vdash e[i] \in_{\infty} \tau
\end{aligned}
$$




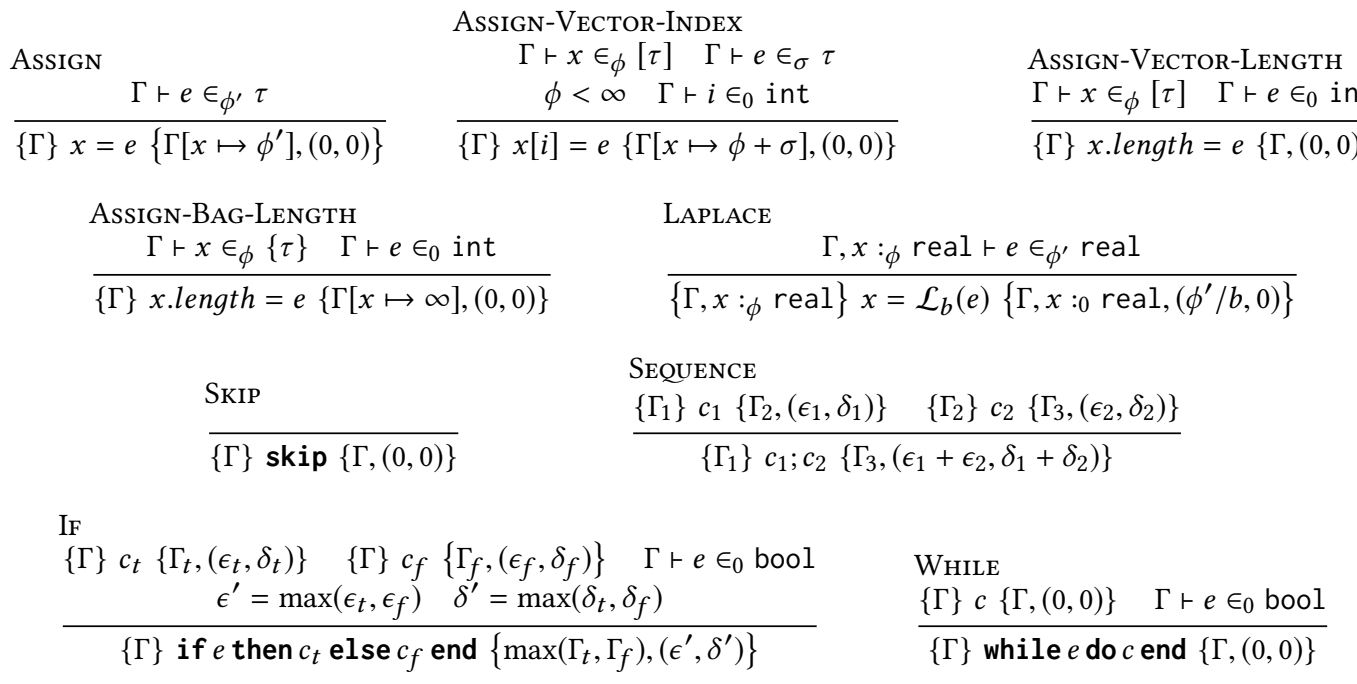

Fig. 3. Core Typing Rules

The Vector-Index rule applies when the lookup expression is 0 -sensitive. A 0 -sensitive index value must be the same across two executions, and the distance between two values at the same position must be bounded by the overall sensitivity of the vector itself according to Definition 3. As an example, given two vectors $[1,2,3]$ and $[1,2,4]$, if we indexed both vectors at the last position, then the resulting values 3 and 4 are at distance 1 apart, which is bounded by the distance between the original vectors. The premise $\phi<\infty$ is necessary to ensure the indexed arrays have the same length in both executions, so that the lookup expression terminates in one execution if and only if it terminates in the other. We refer to this property as co-termination. It is discussed in Section 4.4.

It may be surprising that Fuzzi's typechecker only accepts bag lookup operations over nonsensitive bags. This is due to requirement of co-termination and the fact that bags with non-zero sensitivities may have different lengths in neighboring runs. To see why the bag lookup expression has sensitivity $\infty$, consider two bags $[1,100,2]$ and $[1,2,100]$; these are at distance 0 , but if we access both bags with index 1, the resulting values 100 and 2 are distance 98 apart.

\subsection{Typing Commands}

The typing judgments for commands has the form $\{\Gamma\} c\left\{\Gamma^{\prime},(\epsilon, \delta)\right\}$. We can think of these judgments as a Hoare-triple $-\Gamma$ is a pre-condition of the program $c$, and $\Gamma^{\prime}$ is a post-condition for $c$-annotated with $(\epsilon, \delta)$, the total privacy cost of running $c$.

There are three forms of assignment in Fuzzi: (1) direct assignment to variables, (2) indexed assignment to vectors and bags, and (3) length assignments to vectors and bags. There is a separate typing rule for each form of assignment (Figure 3). The Assign rule updates the LHS variable's sensitivity to the derived sensitivity of the RHS expression. The Assign-Vector-Index rule adds the derived sensitivity of RHS expression to a vector's sensitivity provided the index itself is nonsensitive. (For example, consider the vectors $x s\langle 1\rangle=[1,2,3]$ and $x s\langle 2\rangle=[1,2,4]$. If we perform the assignment $x s[1]=e$ where $e\langle 1\rangle=1$ and $e\langle 2\rangle=10$, then the two vectors become $[1,1,3]$ and $[1,10,4]$, increasing the distance between them by 9 . We require finite sensitivity of the vector variable on the left-hand-side to ensure co-termination-only vectors with finite sensitivities must have the same length.) 
We have separate rules for vector and bag length updates. In the Assign-Vector-Length rule, since the RHS length expression is non-sensitive, the two vectors will be truncated or padded to the same length. In the case of truncation, the distance between the two vectors will decrease or remain the same; on the other hand, if both vectors were padded, since the pad will be the same, they will not introduce any additional distance. Thus, the LHS vector variable's sensitivity remains the same.

In the Assign-BAG-Length rule, it may be surprising that updating the lengths bags-even with a 0 -sensitive new length-can result in $\infty$-sensitivity for the bag. Consider two subsequences of the same length $X$ and $Y$, let $L$ be their identical length. We can choose $X$ and $Y$ such that their bag distance is $2 L$. Now, the two bags $X Y$ and $Y X$ have distance 0 since they contain the same elements, but if we truncated both bags to length $L$, then their distance grows to $2 L$. The Assign-BAG-Length rule must account for this worst case scenario by setting the sensitivity of $x$ to $\infty$.

The core typing rules for operations that involve bags are rather restrictive. We will see in Section 5 how to operate more flexibly over bags using extensions.

The LAPLACE rule computes the privacy cost of releasing a single sensitive real value. The Laplace rule sets the sensitivity of $x$ to 0 after noise is added, which may seem surprising since $x$ 's value is randomized. Intuitively, the 0 -sensitivity expresses that $x$ 's value is now public information and can be used in the clear. We justify 0 -sensitivity as an upper bound on the distance between $x\langle 1\rangle$ and $x\langle 2\rangle$ in Appendix B of the extended version. However, readers do not need to look there to understand the Fuzzi's type sytem design.

The no-op command skip does not alter the program state at all: given any pre-condition $\Gamma$, we can expect the same condition to hold after skip. Also since skip does not release any private data, it has a privacy cost of 0 . This is described by the SKIP rule.

As described in Section 3, the SEQUENCE rule chains together the intermediate $\Gamma$ s for two commands $c_{1}$ and $c_{2}$ and adds up the individual privacy costs for each command.

The control flow command if may modify the same variable with different RHS expressions in each branch; if we allowed expressions with arbitrary sensitivities as the branch condition, we would not be able to derive valid sensitivities for modified variables due to different execution paths. Consider the following example, where $e$ is a sensitive boolean expression: if e then $\mathrm{x}=$ $y$ else $x=z$ end. In one execution, control flow may follow the true branch, assigning $y$ to $x$, while, on the other execution, control flow follows the false branch, assigning $z$ to $x$. Since the typing context $\Gamma$ does not provide any information on the distance between $y$ and $z$, we cannot derive a useful upper bound on $|x\langle 1\rangle-x\langle 2\rangle|$ after the if statement.

On the other hand, if the branch condition is a non-sensitive boolean, then we know that control will go through the same branch in both executions. In this case, we can take the pointwise maximum of the sensitivities from the post-condition of both branches to derive a sound sensitivity for variables modified by the if statement. Similarly, the privacy cost of the entire if statement is bounded by the maximum of the two branches' privacy costs.

The core typing rule for while loops require $\Gamma$ to be a loop invariant of the loop body $c$, the loop guard $e$ a non-sensitive boolean value under $\Gamma$, and the loop body $c$ incur no privacy cost. Had we allowed $e$ be a sensitive boolean, then the while loop may diverge in one execution but terminate in the other. In order to ensure that the two executions co-terminate, we must force the values of $e\langle 1\rangle$ and $e\langle 2\rangle$ before each iteration. We achieve this by checking that the invariant $\Gamma$ of the loop induces a 0 sensitivity on the loop guard $e$.

The Simple Composition Theorem [Dwork et al. 2006] implies that the total privacy cost of a while loop is bounded by the sum of individual privacy cost from each iterations. Even though we can ensure the two executions of while loops co-terminate, we cannot always statically tell for how many iterations both loops will run. In order to ensure the soundness of the total privacy cost 
estimation, we conservatively forbid loop bodies from using any commands that will increase $\epsilon$ or $\delta$.

These core typing rules place rather heavy restrictions on Fuzzi programs operating over vectors and bags, or programs using conditionals and loops; for example, the core rules grossly overestimate sensitivities for vectors and bags and forbid sensitive booleans in branch and loop conditions. The core rules are designed for chaining together blocks of differentially private mechanisms, and these typing rules are often not enough to typecheck the implementation of interesting differentially private algorithms. We will see how to teach Fuzzi's typechecker to derive more precise sensitivities for differentially private mechanisms involving all these constructs in Section 5.

\subsection{Soundness}

There has been a rich line of work on developing type systems and language safety properties using foundational methods [Ahmed 2006; Appel and McAllester 2001; Appel et al. 2007; Frumin et al. 2018; Jung et al. 2017, etc.]. The foundational approach develops the typing rules of a language as theorems in an expressive logic. Type systems developed using foundational methods benefit from the soundness of the underlying logic: if a typing rule is proven true as a theorem, then adding new rules as theorems to the type system will not break validity of existing rules. Most importantly, the foundational approach allows Fuzzi to mix typing rules for an automated typechecker with specialized typing rules extracted from manual proofs of differential privacy.

We choose apRHL [Barthe et al. 2016] as the foundational logic to build Fuzzi's type system upon. The apRHL logic extends Floyd-Hoare Logic [Hoare 1969] with relational assertions, reasoning of probabilistic commands, and differential privacy cost accounting. An apRHL judgment has the form $\vdash c_{1} \sim_{(\epsilon, \delta)} c_{2}: \Psi \Rightarrow \Phi$. The metavariables $c_{1}$ and $c_{2}$ stand for two programs related by this judgment, the annotations $\epsilon$ and $\delta$ stand for the quantitative "cost" of establishing this relation, and $\Psi$ and $\Phi$ are both assertions over pairs of program states, standing for the pre-condition and the post-condition of this judgment respectively.

We have seen two kinds of rules in the Fuzzi type system so far: expression typing rules and core typing rules for commands. Although both are presented in the form of inference rules, these two typing judgments are very different in nature. The expression typing rules are defined as an inductive relation, while the typing rules for commands are theorems to be proven. This choice is motivated more by practicality and less by theory-foundational proofs are more difficult to work with than inductive relations, since we do not plan on mixing the typing rules for expressions with manual proofs, there is no need to use the foundational methods for expressions.

Because expression typing rules of the form $\Gamma \vdash e \epsilon_{\phi} \tau$ are instances of an inductive relation, we need to prove a few soundness properties that will make these expression typing rules useful in the development of command typing rule proofs. In particular, we care about soundness with respect to sensitivity and co-termination. We elide proofs by straightforward induction.

Lemma 1 (Expression Sensitivity Sound). Given $\Gamma \vdash e \in_{\phi} \tau$ and two program states $M_{1}$ and $M_{2}$ related by $\Gamma$, if $\llbracket e \rrbracket M_{1}=v_{1}$ and $\llbracket e \rrbracket M_{2}=v_{2}$, then $d_{\tau}\left(v_{1}, v_{2}\right) \leq \phi$.

LEMMA 2 (EXPRESSION Co-TERMINATION). Given $\Gamma \vdash e \in_{\phi} \tau$ and two program states $M_{1}$ and $M_{2}$ related by $\Gamma$, evaluating the expression $\llbracket e \rrbracket M_{1}$ yields some value $v_{1}$ if and only if $\llbracket e \rrbracket M_{2}$ yields some value $v_{2}$.

The command typing rules have the form $\{\Gamma\} c\left\{\Gamma^{\prime},(\epsilon, \delta)\right\}$. And earlier, we described $\Gamma$ and $\Gamma^{\prime}$ as pre-condition and post-conditions. What does it mean to treat a typing context as pre- and post-conditions?

Recall the translation from typing contexts to apRHL assertions in Section 3. This translation naturally induces a relation on each program variable: each variable's type information $x:_{\phi} \tau$ 
becomes the relation $d_{\tau}(x\langle 1\rangle, x\langle 2\rangle) \leq \phi$. The entire typing context $\Gamma$ is translated to conjunctions of the pointwise relation for each program variable. As an example, the context $x:_{1}$ int, $y:_{2}$ real corresponds to the relation $d_{\text {int }}(x\langle 1\rangle, x\langle 2\rangle) \leq 1 \wedge d_{\text {real }}(y\langle 1\rangle, y\langle 2\rangle) \leq 2$. We also use the $\llbracket \cdot \rrbracket$ function to denote the translation of typing contexts.

So a typing rule is in fact an apRHL judgement in disguise: $\vdash c \sim_{(\epsilon, \delta)} c: \llbracket \Gamma \rrbracket \Rightarrow \llbracket \Gamma^{\prime} \rrbracket$. To prove these judgments valid, we need to use the apRHL proof rules. In fact, many of Fuzzi's core typing rules are specialized versions of the corresponding apRHL rule for that command. We list all apRHL proof rules used in this paper in Appendix B of the extended version, but readers do not need to look there to understand the following content in the main body of the paper.

As an example, the soundness of the Assign rule is justified by the following lemma:

Lemma 3. Given $\Gamma \vdash e \epsilon_{\phi^{\prime}} \tau$, the judgement $\vdash x=e \sim_{(0,0)} x=e: \llbracket \Gamma \rrbracket \Rightarrow \llbracket \Gamma\left[x \mapsto \phi^{\prime}\right] \rrbracket$ is true.

We define one such lemma for each of the typing rules given in Figure 3, and justify them using corresponding apRHL proof rules.

One important technical subtlety is that the original presentation of apRHL only reasons over terminating programs. Requiring Fuzzi's typechecker to prove termination for all programs would unavoidably rule out some useful ones. Fortunately, we actually need only a subset of apRHL's proof rules, and these are all sound even if programs only co-terminate [Hsu 2018]; we can thus we relax the "all programs terminate" assumption of apRHL in the development of Fuzzi.

Remark. Although we carry out privacy proofs in apRHL, the logic apRHL does not fully isolate its user from the underlying semantics of the language. For example, some of the apRHL proof rules used to develop Fuzzi require us to prove termination of commands, but apRHL does not give proof rules for termination. So we develop our own sound termination typing rules that match apRHL's termination definition, using the semantics of Fuzzi. This necessitates an even lower-level logic $\mathcal{L}$ to formalize the parts not specified by apRHL. In the following sections, we will explicitly call out objects defined in $\mathcal{L}$. $^{2}$

\section{EXTENSIONS}

In this section, we discuss how to integrate the core Fuzzi type system with specialized typing rules for Fuzzi extensions; we then introduce several concrete extensions that will be used later for our case studies: operations for mapping a piece of code over all the cells in a bag or vector, an operation for partitioning a bag into a collection of smaller bags according to some criterion, an operation for summing the elements of a bag, and an operation for sequencing several commands using an "advanced composition theorem" from the differential privacy literature to obtain a lower privacy cost than the one given by the plain sensitivity typing rule for sequencing.

Definition 5. An extension is a 4-tuple (ext, $f$, rule, proof). The first field ext is the name of the extension. The second field is a function $f$ that maps Fuzzi expressions or commands to a Fuzzi command, we will call $f$ the syntax expansion function. Let $v_{1}, \ldots, v_{i}$ be the syntactic variables bound in $f$; the third field is a typing rule, parameterized by the same $v_{1}, \ldots, v_{i}$ syntactic variables. The typing rule may contain premises over any combination of $v_{1}, \ldots, v_{i}$, and the typing rule's conclusion has the shape of a Fuzzi typing triple for the expanded code of the extension. Finally, the last field proof is a proof of the soundness of the typing rule.

We will use the notation $\operatorname{ext}\left(p_{1}, p_{2}, \ldots, p_{i}\right)$ for the syntax of invoking an extension. These extension commands are replaced by the expanded body $f\left(p_{1}, p_{2}, \ldots, p_{i}\right)$ with each $p_{i}$ substituting for each syntax variable $v_{i}$.

\footnotetext{
${ }^{2}$ The proof assistant Coq [Coq Development Team 2018] is a suitable candidate of $\mathcal{L}$; indeed, we have already formalized some parts of Fuzzi in Coq.
} 

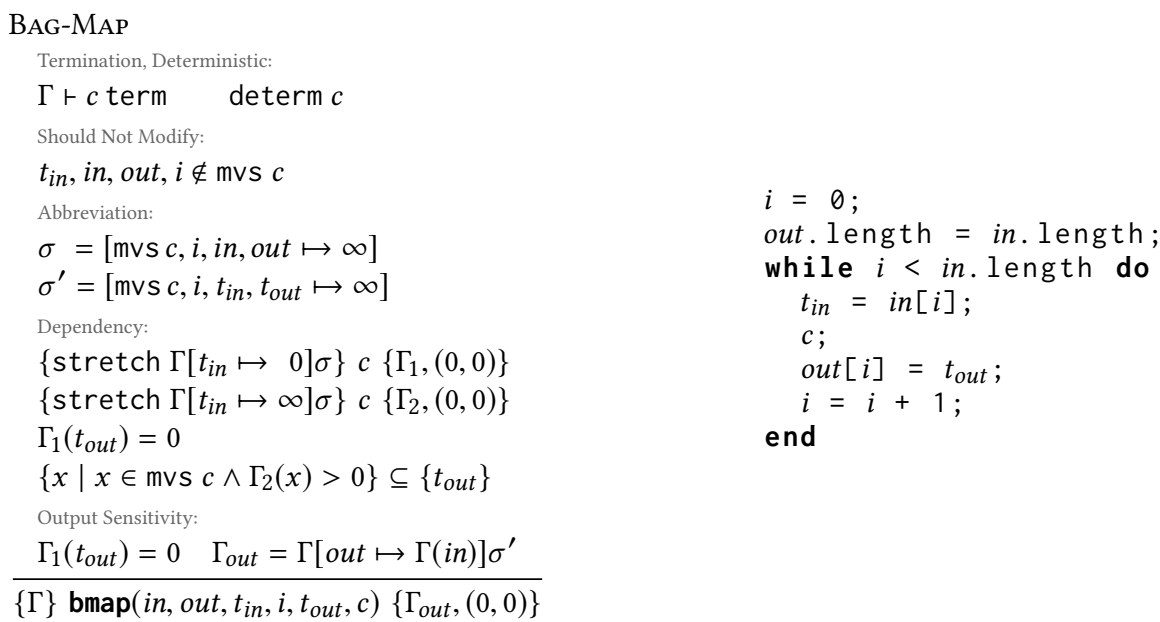

Fig. 4. BAG-MAP typing rule and extension code pattern

Expressing the typing rule requires the full generality of the lowest level logic $\mathcal{L}$, since extension typing rules contain side conditions (such as termination) that are not captured by apRHL. The general shape of this theorem is of the form

$$
\forall v_{1}, \ldots, v_{i}, P_{1} \wedge \cdots \wedge P_{j} \Rightarrow\{\Gamma\} \operatorname{ext}\left(v_{1}, \ldots, v_{i}\right)\left\{\Gamma^{\prime},(\epsilon, \delta)\right\}
$$

Each $P_{j}$ is a premise of the typing rule, and all premises may bind any combination of $v_{1}, \ldots, v_{i}$. The conclusion of the theorem is always of the shape of a Fuzzi typing triple, so that proofs of the typing rule can mix with the soundness proofs of the core typing rules introduced in Section 4.1. The final component of an extension is a soundness proof of the typing rule theorem.

Some of these premises are Fuzzi typing judgments, while some others are auxiliary judgments that assert termination or describe a linear scaling relationship between the pre-condition sensitivities and the post-condition sensitivities. These two extra kinds of auxiliary judgments are defined in $\mathcal{L}$, we will give definitions for these auxiliary judgments as we encounter them. We will describe their proof rules in Appendix $\mathrm{C}$ of the extended version.

We will provide an overview of the use case for each extension, and only provide a sketch of the proof of soundness to conserve space. Detailed soundness proofs for all the extensions can be found in Appendix D of the extended version.

\subsection{Bag Map}

Our first extension, BAG-MAP, takes an input bag variable, an output bag variable, a few auxiliary variables used by the expanded loop, and finally a "bag-map body" $c$ that reads from a single bag entry and outputs a mapped value for that bag entry. This command $c$ represents a single step of the "map" operation. BAG-MAP applies this operation uniformly for all entries in a bag.

The premises of BAG-MAP's typing rule use a few new ingredients-the function mvs collects the set of modified variables from a command $c$, and the function stretch "expands" a typing context. The stretch function takes a typing context $\Gamma$, and for each variable $x \in \Gamma$, if $\Gamma(x)>0$, sets $\Gamma(x)=\infty$, otherwise leaves $\Gamma(x)$ as 0 . 


$$
\begin{array}{ll}
\text { stretch } \varnothing & =\varnothing \\
\text { stretch }\left(x:_{0} \tau, \Gamma\right) & =x:_{0} \tau \text {, stretch } \Gamma \\
\text { stretch }\left(x:_{\sigma} \tau, \Gamma\right) & =x:_{\infty} \tau \text {, stretch } \Gamma \quad \text { if } \sigma>0
\end{array}
$$

The function stretch is used when we need to verify that some output variable's sensitive value derives solely from some input variable. ${ }^{3}$

Since a variable can only become sensitive if its value derives from another sensitive variable, it is perhaps not surprising that Fuzzi's sensitivity type system is capable of tracking dependency as well. We will use the function stretch to help uncover this dependency analysis part of the sensitivity type system.

Consider any program fragment $c$, for which we want to verify that for a single variable $t$, after executing $c$, the sensitive data held in $t$ must only come from $s$. If we had the following typing judgment about $c$ : $\left\{\right.$ stretch $\left.\Gamma_{1}[s \mapsto 0]\right\} c\left\{\Gamma_{2},(0,0)\right\}$ where $\Gamma_{2}(t)=0$, then we know if $s$ is non-sensitive, $t$ is also non-sensitive. This implies that the only sensitive dependency of $t$ is at most the singleton set $\{s\}$.

However, this typing judgment only tells us the dependency of $t$ when all sensitive variables are $\infty$-sensitive before executing $c$. Does the same result hold when those variables have finite sensitivity? To arrive at this conclusion, we need to apply the CONSEQ rule from apRHL after unfolding our previous typing judgment into an apRHL judgement.

$$
\begin{aligned}
& \text { CONSEQ } \\
& \vdash c_{1} \sim \epsilon^{\prime}, \delta^{\prime} c_{2}: \Phi^{\prime} \Rightarrow \Psi^{\prime} \quad \vDash \Phi \Rightarrow \Phi^{\prime} \\
& \vDash \Psi^{\prime} \Rightarrow \Psi \quad \vDash \epsilon^{\prime} \leq \epsilon \quad \vDash \delta^{\prime} \leq \delta \\
& \hline \vdash c_{1} \sim(\epsilon, \delta) \\
& \hline c_{2}: \Phi \Rightarrow \Psi
\end{aligned}
$$

The ConseQ rule allows us to strengthen the pre-condition. Now, in the context $\operatorname{stretch} \Gamma_{1}[s \mapsto 0]$, if we change any other variable's sensitivity to some finite value, then we have a stronger statement, since $\infty$-sensitivity is implied by finite sensitivity. Thus, the ConseQ rule allows us to change the pre-condition to one that implies the stretched typing context.

This technique allows us to verify $t$ 's dependency is at most $\{s\}$ through sensitivity analysis. However, the program $c$ may modify variables other than $t$. In order to make sure there are no other sensitive output variables from the program fragment $c$, we also want to verify the lack of dependency on $s$. Can we re-use the sensitivity type system to check some other modified variable $v$ does not depend on the variable $s$ ? Indeed we can, using the stretch function again in a slightly different way. Consider the typing judgment $\left\{\right.$ stretch $\left.\Gamma_{1}[s \mapsto \infty]\right\} c\left\{\Gamma_{2},(0,0)\right\}$ where $\Gamma_{2}(v)=0$ This typing judgment tells us that no matter how much $s$ and the other sensitive values changes between two executions of $c$, the value held in $v$ remains the same at the end of the execution. So indeed $v$ does not depend on $s$ or any other sensitive variable. Again ConsEQ rule allows us to strengthen the $\infty$-sensitivity to any finite sensitivity.

The BAG-MAP typing rule applies this technique to check that, on each iteration of $c$, the value of $t_{\text {out }}$ derives only from the corresponding input bag entry $t_{\text {in }}$. The program fragment $c$ should not access the original bag value directly, and neither should it write directly to the output bag. Furthermore, each iteration of the bag map body should be independent of each other, so the values of its modified variables should not carry over to the next iteration. For these reasons, we set the sensitivity of modified variables of $c$, the variable $i$, and the input and outputs bags variables in and out to $\infty$ in the judgment $\left\{\right.$ stretch $\left.\Gamma\left[t_{\text {in }} \mapsto 0\right] \sigma\right\} c\left\{\Gamma_{1},(0,0)\right\}$, and check $\Gamma_{1}\left(t_{\text {out }}\right)=0$. We use the letter $\sigma$ to abbreviate the update expression [mvs $c, i$, in, out $\mapsto \infty]$.

\footnotetext{
${ }^{3}$ This kind of dependency analysis is one of the motivating examples for Benton's seminal work on relational Hoare logic [Benton 2004].
} 
We also want to verify that none of the modified variables, except for $t_{\text {out }}$, has any dependency on sensitive data. This is why we check the only variable that can potentially hold sensitive data is $t_{\text {out }}$ in the judgment $\left\{\right.$ stretch $\left.\Gamma\left[t_{\text {in }} \mapsto \infty\right] \sigma\right\} c\left\{\Gamma_{2},(0,0)\right\}$ The variables mvs $c$, i, in, out are again set to $\infty$ to ensure these variables do not leak information across iterations as discussed above.

We use the judgment determ $c$ to assert that $c$ is a deterministic Fuzzi program. It is easy to show that any program $c$ that do not contain the Laplace mechanism is deterministic. We use the judgment $\Gamma \vdash c$ term to denote that command $c$ terminates for any program state that is well-typed according to shape $(\Gamma)$. That is, for all $M$ in shape $(\Gamma)$, running the program $c$ with the well-typed program state $\llbracket c \rrbracket M$ terminates with probability 1 .

Since the apRHL judgment that corresponds to the conclusion of this typing rule implies cotermination of BAG-MAP programs, we need to prove the expanded while loops actually co-terminate. However, because these two while loops may have different number of iterations due to bags having different sizes, thus executing $c$ for different number of times, we cannot simply show $c$ co-terminates. So, we take the extra step of requiring termination of $c$ on all well-shaped inputs, which ensures both loops will always terminate.

The soundness proof for the BAG-MAP typing rule applies dependency analysis to ensure the map body $c$ maps the input value $t_{\text {in }}$ deterministically to $t_{\text {out }}$, and that $c$ does not store sensitive data in any of its other modified variables. This allows us to ensure in $[i]$ maps deterministically and uniformly to out $[i]$ through each iteration of $c$. Now, adding or removing any entry from the input bag will correspondingly add or remove the mapped value from the output bag. So the output bag must have the same sensitivity as the input bag.

\subsection{Vector Map}

Our second example, the Vector-MAP extension is, very similar to BAG-MAP in that it also requires the "map" command to restrict its flow of sensitive data from $t_{\text {in }}$ to only $t_{\text {out }}$. However, Vector-MAP has an additional requirement that map body must be "linear":

Definition 6 (Linear Commands). We write $k \Gamma$ to denote a typing context $\Gamma^{\prime}$ where $\Gamma^{\prime}(x)=k \Gamma(x)$. A deterministic and terminating command $c$ is linear with respect to $\Gamma_{1}$ and $\Gamma_{2}$, if for any $k>0$, the scaled typing judgment $\left\{k \Gamma_{1}\right\} c\left\{k \Gamma_{2},(0,0)\right\}$ is true. We define $k \cdot \infty=\infty$ for $k>0$, and $0 \cdot \infty=0$.

This definition tells us that the updates in sensitivity in the post-condition scale linearly with respect to the sensitivities in the pre-condition. An example of a linear command is $x=2 y+1$ with typing context $\Gamma_{1}=x:_{0}$ real, $y:_{1}$ real and $\Gamma_{2}=x:_{2}$ real, $y:_{1}$ real.

A counterexample is if $x>0$ then $x=x+1$ else $x=x+2$ end with $\Gamma_{1}=x: 1$ real and $\Gamma_{2}=x: 2$ real. This command conditionally increments $x$ by a constant of 1 or 2 , so if $x$ was 1 sensitive before executing this command, then we can show in apRHL that $x$ is 2 sensitive after this conditional command. Now, if we scaled $x$ 's sensitivity by 0.5 , then $x$ is 1.5 sensitive after this conditional increment, rather than $2 \cdot 0.5=1$. So this command is not linear with respect to the chose $\Gamma_{1}$ and $\Gamma_{2}$. However, had we chosen $\Gamma_{2}=x:_{\infty}$ real, then this command is linear with respect to the new post-condition, because $k \cdot \infty=\infty$, and no matter what the values of $x\langle 1\rangle$ and $x\langle 2\rangle$ are, their difference is always bounded by $\infty$. We present the proof rules for linear commands in Appendix $\mathrm{C}$ of the extended version.

For vector map, we need to know the scaling relationship between the sensitivity of $t_{\text {out }}$ and the sensitivity of $t_{\text {in }}$ in order to derive the sensitivity of the output vector. With $c$ being a linear command for the chosen pre-condition $\Gamma\left[t_{\text {in }} \mapsto 1\right] \sigma$ and post-condition $\Gamma_{3}$, by the definition, for any scale factor $k>0$ we know if $d\left(t_{\text {in }}\langle 1\rangle, t_{\text {in }}\langle 2\rangle\right) \leq k$ before executing $c$, then $d\left(t_{\text {out }}\langle 1\rangle, t_{\text {out }}\langle 2\rangle\right) \leq s k$ after executing $c$, where $s=\Gamma_{3}\left(t_{\text {out }}\right)$. Recall the definition of vector distance, by instantiating $k$ with the actual distance for each pair of $i$ th entries from the input vectors $d(i n\langle 1\rangle[i], i n\langle 2\rangle[i])$, we know 

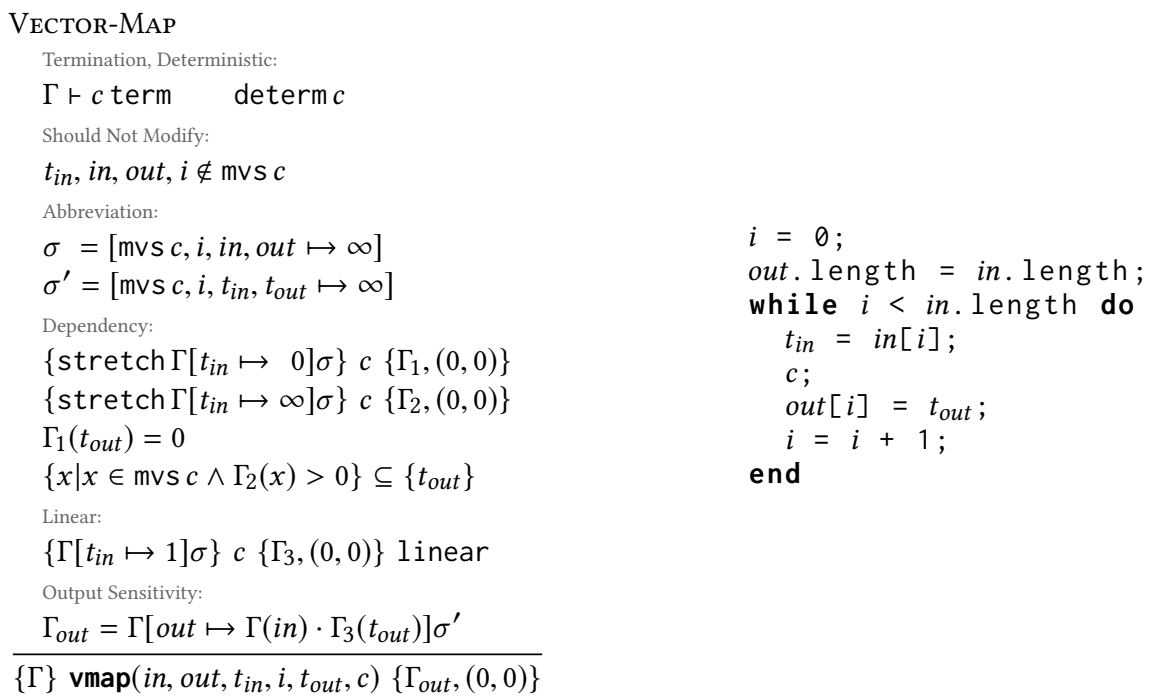

Fig. 5. VeCtoR-MAP typing rule and extension code pattern

the distance between the output vectors satisfy the following condition:

$$
\begin{aligned}
d_{[\tau]}(\text { out }\langle 1\rangle, \text { out }\langle 2\rangle) & =\sum_{i} d_{\tau}(\text { out }\langle 1\rangle[i], \text { out }\langle 2\rangle[i]) \\
& \leq \sum_{i} s d_{\tau}(\operatorname{in}\langle 1\rangle[i], \operatorname{in}\langle 2\rangle[i]) \\
& =s d_{[\tau]}(\operatorname{in}\langle 1\rangle, \operatorname{in}\langle 2\rangle) .
\end{aligned}
$$

This justifies the sensitivity derived by the typing rule for vector map.

\subsection{Partition}

Our third extension, PARTition, allows programmers to break apart a larger bag into a vector of smaller bags. PARTITION is parameterized by an input bag variable, an output vector variable, a few auxiliary variables for storing results from intermediate computations, and finally a command that maps each input bag entry to a partition index.

The PARTITION extension is similar to BAG-MAP in that it maps each bag item to some value, but they differ in how the output value from the each iteration is used. With BAG-MAP, the output value from each iteration is collected into the output bag as is. PARTition uses the output value as an assignment index into the output bag out, and appends the bag entry at current iteration to the sub-bag at out $\left[t_{i d x}\right]$. As an example, if the input bag is $[1.2,2.3,3.4]$, and the map operation simply rounds down each value to the nearest integer, then the output bag will be [[], [1.2], [2.3], [3.4]].

It may seem redundant that PARTITION takes the number of partitions as a parameter. Shouldn't PARTITION be able to compute the number of partitions as it processes the input bag values? It should not, because a computed number of partitions is a sensitive value that depends on the contents of the input bag. Taking the previous example, if we add a value of 100.1 to the input bag and do not fix the number of partitions ahead of time, then the output vector will have 97 more sub-bags than the original output vector-i.e., the distance between the output vectors can be 


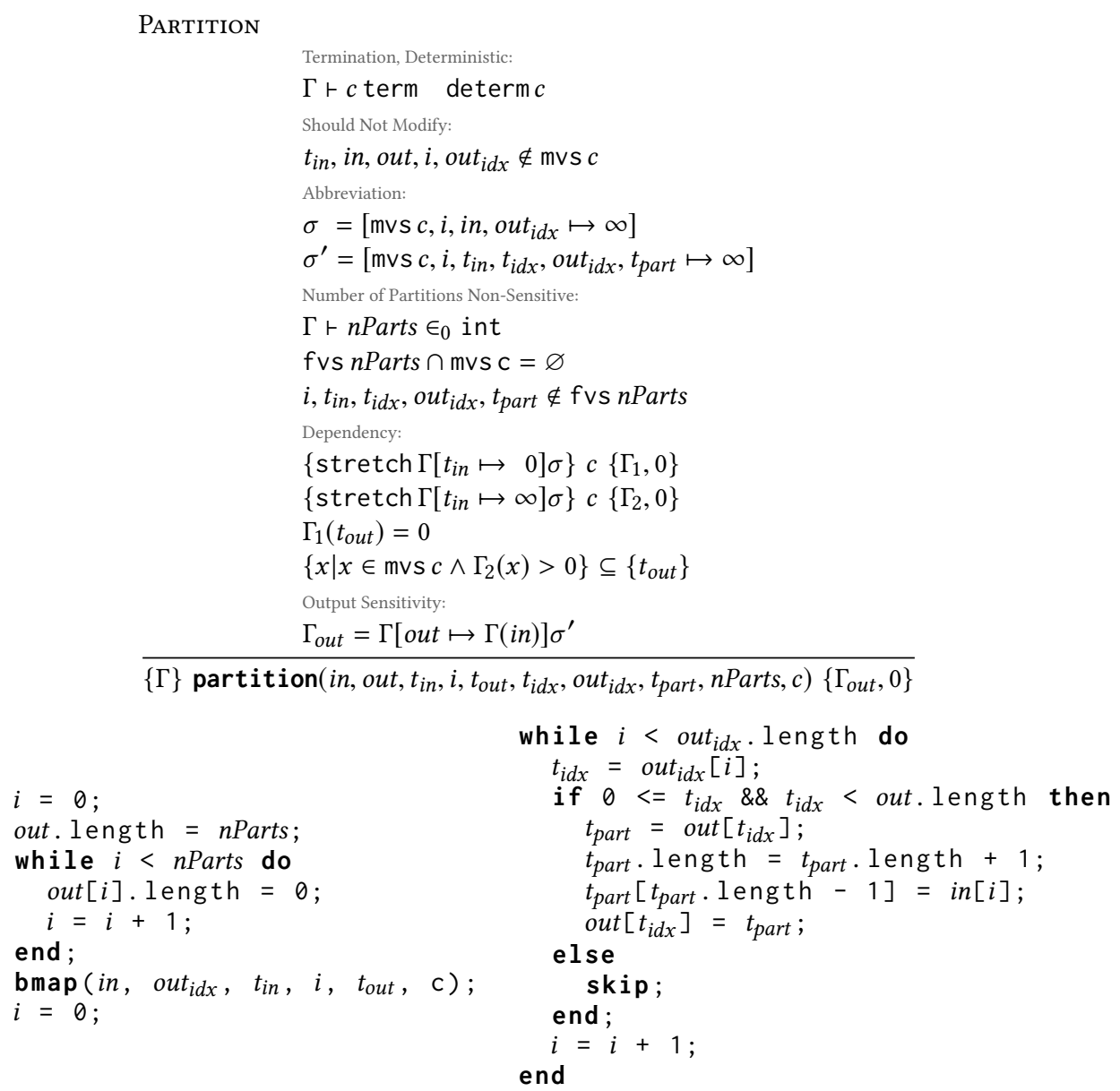

Fig. 6. PARTITION typing rule and extension expansion

made arbitrarily large by adding a single item to the input bag. This is why we fix the number of partitions and drop the items whose partition indices are out of range.

The soundness of the sensitivity check for partition comes from the fact that each index is derived only from its corresponding bag entry, thus adding or removing one bag entry can cause at most one sub-bag in the output vector to vary by distance 1 . Generalizing this fact shows that the output vector has the same sensitivity as the input bag does to partition.

\subsection{Bag Sum}

Our fourth extension, BAG-Sum, works with bags of real-valued data and adds these values up with clipping. The clipping process truncates a value $s$ such that its magnitude is no larger than bound. This is important to ensure the output of BAG-Sum has finite sensitivity. Recall that the sensitivity definition on a bag places no constraints on the distance of values held by the bag. If we naïvely summed the two bags $[1,2]$ and $[1,2,100]$, although their bag distance is bounded by 1 , their sums have distance 100. Using only the bag distance, the typechecker will have no information on the sensitivity of the sum. Truncating each value into the range [-bound, bound] allows us to bound 


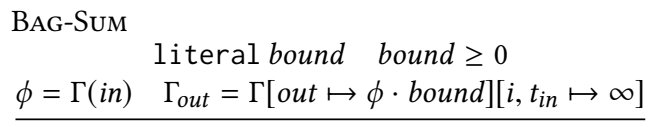

$\{\Gamma\} \operatorname{bsum}\left(\right.$ in, out $, i, t_{\text {in }}$, bound $)\left\{\Gamma_{\text {out }}, 0\right\}$

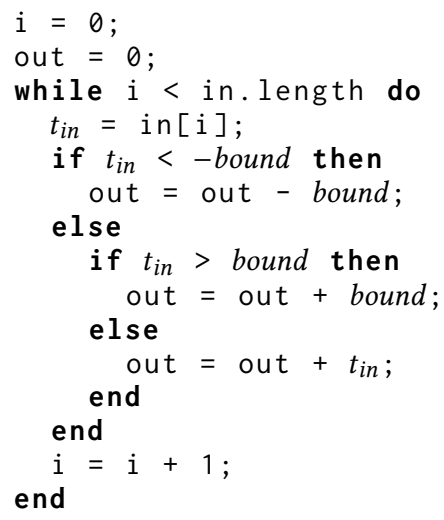

Fig. 7. BAG-SUm typing rule and expansion
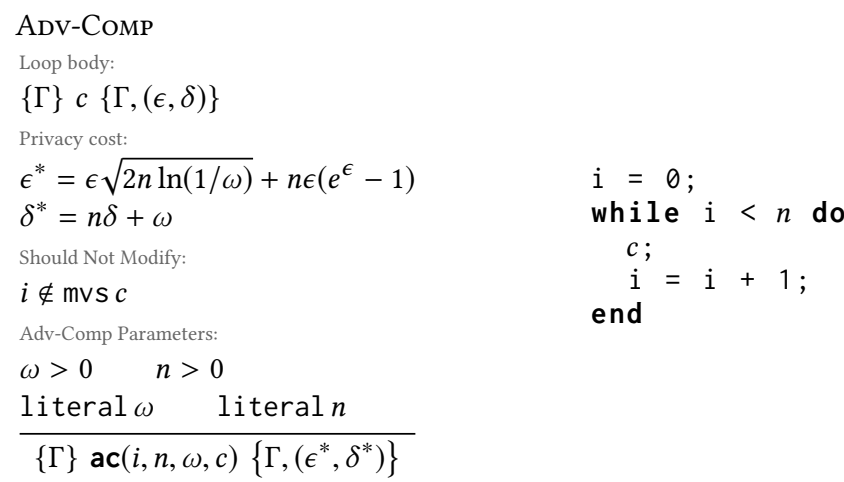

Fig. 8. Adv-Comp typing rule and expansion

the total sensitivity of the sum value-if up to $\phi$ bag items may be added or removed, and each can contribute up to bound towards the total sensitivity of out, then at the end of the loop, out must be $(\phi \cdot$ bound $)$-sensitive.

\subsection{Advanced Composition}

Our fifth extension, Adv-Comp, simply expands to a loop that runs the supplied command $c$ for $n$ times. However, this extension provides a special privacy cost accounting mechanism known as Advanced Composition [Dwork et al. 2010]. Compared to Simple Composition, ADv-Comp gives an asymptotically better $\epsilon$ that grows at the rate of $O(\sqrt{n})$, at the cost of a small increase in $\delta$. Simple composition of loop iterations will give privacy costs that grow at the rate of $O(n)$ instead. The programmer chooses the increase in $\delta$ by providing a positive real number $\omega$, which is used to compute the aggregated privacy cost for the entire loop.

The ADV-Comp extension is useful for programs that iteratively release data, and it also allows programs to be run for more iterations while staying under the same privacy budget. We use ADV-Comp in our implementation of logistic regression in Section 7.

It is worth noting that ADV-Comp does not always give a better privacy cost than simple composition: when the $\epsilon$ cost of $c$ is large, the term $n \epsilon\left(e^{\epsilon}-1\right)$ becomes the dominating term. This 
term again grows linearly with $n$, and it has a multiplicative factor of $e^{\epsilon}-1$. When ADV-Comp gives worse privacy cost in both $\epsilon$ and $\delta$ in comparison to simple composition, the type system falls back to simple composition for the expanded loop for privacy cost accounting.

\section{IMPLEMENTATION}

Our prototype Fuzzi checker expands extensions before typechecking, leaving hints in the expanded abstract syntax tree so that it can tell when to apply the macro-typing rules that accompany each extension. The typechecking algorithm includes three major components: 1) a checker that computes sensitivity, 2) a checker for termination, and 3) a checker for linear properties of commands.

The implementation uses three separate ASTs types-called IMP, IMPExT, and IMPTC-to represent a Fuzzi program in different phases of checking. The IMP AST is what the parser produces-i.e., the constructs of the core language plus extension applications. IMPExT is a convenient language for entering extension declarations for Fuzzi. Finally, IMPTC represents programs expanded from IMP -while-language with no extension application nor extension definition, but contains typechecker hints, and the typechecker expects terms from IMPTC. The IMPTC language is not accepted by the parser, as we do not anticipate users wanting to enter typechecker hints directly. We use the extensible sum encoding described in data types à la carte [Swierstra 2008] to represent these ASTs in order to avoid code duplication. We depend on the compdata package [Bahr and Hvitved 2011] to manipulate ASTs in this encoding.

The three checkers are implemented separately. A checker composition function takes results from each checker, and produces the final type information for a Fuzzi program.

To efficiently execute Fuzzi code, we compile Fuzzi programs to Python and use fast numeric operations from the numpy library [Oliphant 2015] whenever appropriate.

\section{EVALUATION}

To evaluate Fuzzi's effectiveness, we implement four differentially private learning algorithms from four diverse classes of learning methods-discriminative models, ensemble models, generative models, and instance-based learning. The algorithms and datasets are both taken from canonical sources. We want to know (1) whether Fuzzi can express these algorithms adequately, (2) whether the typechecker derives sensitivity bounds comparable to results of a careful manual analysis, and (3) whether the final privacy costs are within a reasonable range.

We use datasets obtained from the UCI Machine Learning repository [Dheeru and Karra Taniskidou 2017] and the MNIST database of handwritten digits [LeCun and Cortes 2010]. We focus on evaluating Fuzzi's usability on prototyping differentially private learning tasks in these experiments, rather than trying to achieve state-of-the-art learning performance.

We find that Fuzzi can indeed express all four examples and that it correctly derives sensitivity bounds comparable to results from a manual analysis. The examples also demonstrate that the extensions described in Section 5 are useful for real-world differential privacy programming, since each of the learning algorithms can be expressed as a straightforward combination of extensions.

On the other hand, the privacy costs that Fuzzi derives are arguably a bit disappointing. One reason for this is that we ran the experiments on fairly small datasets. A deeper reason is that Fuzzi focuses on accurate automatic inference of sensitivities, an important building block in differential privacy. Tracking sensitivities is somewhat orthogonal to the question of how to most tightly track privacy costs, which is achieved via composition theorems that sit on top of the sensitivity calculations. Our focus in this work has been mainly on tracking sensitivity; in particular, we implement only simple composition theorem in the core type system. The result is that Fuzzi may report a larger privacy cost than is optimal, even when it optimally computes sensitivities. However, stronger composition theorems can be added as extensions: we give an example of this

Proc. ACM Program. Lang., Vol. 3, No. ICFP, Article 93. Publication date: August 2019. 
by demonstrating an "advanced composition" [Dwork et al. 2010] extension in Section 5. We view adding extensions for more sophisticated methods of tracking privacy costs as future work (see Section 9 and Section 10).

\subsection{Logistic Regression}

We first investigate a binary classification problem. The dataset contains 12,665 digits (either 0 or 1 ) from the MNIST database. (We only work with 0 and 1 digits because it simplifies our presentation. A 10-class logistic regression model that classifies all 10 digits can be implemented using the same methods we show here.) We use 11,665 digits for training and leave 1,000 digits on the side for evaluation. Each digit is represented by a $28 \times 28$ grayscale image plus a label indicating whether it is a 0 or a 1 . The image and its label are flattened into a 785 -dimensional vector. We then use gradient descent, a simple and common machine learning technique to train a standard logistic regression model that classifies these two shapes of digits. We apply differential privacy here to protect the privacy of each individual image of the digits. In other words, differential privacy limits an adversary's ability to tell whether a particular image was used in training the classification model. In particular, we modify gradient descent with a gradient clipping step to achieve differential privacy. Gradient clipping is a common technique for implementing differentially private gradient descent [Abadi et al. 2016; McMahan et al. 2018].

The logistic regression model is parameterized by a vector $\vec{w}$ of the same dimension as the input data and a scalar $b$. A "loss function" $L\left(\vec{w}, b, \vec{x}_{i}\right)$ quantifies the mistakes the model makes given a pair of $\vec{w}$ and $b$, and an input image $x_{i}$. In ordinary (non-private) gradient descent, we compute the gradients $\frac{\partial L}{\partial \vec{w}}$ and $\frac{\partial L}{\partial b}$ for each image $x_{i}$, and we move the current parameters $\vec{w}$ and $b$ in the direction of the average of these gradients, decreasing the value of the loss function $L$ (i.e., improving the quality of model parameters). To set the initial values of $\vec{w}$ and $b$, we take random samples from a normal distribution centered at 0 and with variance 1.

Since the gradients here are computed from private images, the model parameters modified with these gradients are also sensitive information that cannot be released directly. Instead, we release noised estimations of the average gradients and use these values to update model parameters. We apply bmap over the input dataset, computing a bag of both gradients for each image. We then use bsum and the Laplace mechanism to release the sum of the gradients. We also use the Laplace mechanism to compute a noised estimate of the size of the dataset, and then update the model parameter with the noised average gradient. The bsum extension clips each gradient value so that the final sum has a bounded sensitivity.

We iterate the gradient descent calculation with the ac (advanced composition) extension. With 100 passes over the training set, we reach a training accuracy of 0.933 , and test accuracy of 0.84 . We measure accuracy as the fraction of images the trained model correctly classifies. The differentially private model's accuracy is comparable to the accuracy of 0.88 for a logistic regression model without differential privacy [Lecun et al. 1998]. Training the model with 100 passes incurs privacy cost $\epsilon=11.02$ and $\delta=10^{-6}$. Our $\epsilon$ privacy cost is larger than the results achieved by Abadi et al. [2016] $\left(\epsilon=2.55, \delta=10^{-5}\right)$ on MNIST, due to our use of a simpler privacy composition theorem; Abadi et al. invented a specialized "moments accountant" method to derive tighter aggregated privacy costs given the same sensitivity analysis.

\subsection{Teacher Ensemble}

Next, we build on the logistic regression from above, together with ideas from Papernot et al. [2016], to design an ensemble model-a collection of models-that classifies 0 and 1 digits from the MNIST dataset. Papernot et al. demonstrated a general approach for providing privacy guarantees with respect to the training data: first partition the private training data into $k$ partitions, then apply a 
training procedure to each partition to build a private model for it. These $k$ private models form a Private Aggregation of Teacher Ensembles (PATE). The PATE then predicts labels using differential privacy for another unlabeled public data set. Since the resulting public dataset and its labels are not private information, they can be used to train any other model, effectively transferring knowledge from the PATE to a public model while preserving privacy. Note that we do not require that the models used internally in creating the PATE to be differentially private: only the aggregation step (used to predict labels for the public dataset) involves differential privacy.

We split the training input dataset of MNIST digits into a bag of five parts. Using the extension bmap for each part, we independently train a logistic regression model with non-private gradient descent. Assuming the input dataset has sensitivity 1, only one part of training data can change. Fuzzi correctly derives the fact that therefore at most 1 trained logistic regression model will change, resulting in a bag of model parameters with sensitivity 1 .

We use the private ensemble of models to label another 100 test images; with privacy cost $\epsilon=20.0$, and $\delta=0.0$, we are able to reach an accuracy of 0.82 . The large $\epsilon$ value here is related to the small size of the training set. To release a public label for a given image, the private scores are collected from the PATE with bmap and then a noised average of the private scores is released by bsum with the Laplace mechanism. Since we only have 5 private scores for each image, the noise variance must be small so as not to destroy the utility of the scores, resulting in big $\epsilon$. To increase the stability of the released label (and hence decrease the privacy cost) we could increase the number of models, thus increasing the number of private scores and the scale of the summed score, thereby allowing more noise added to their average. However, the result would be that each model would have been trained on correspondingly fewer images, resulting in worse classification performance on this dataset. On larger datasets, our Fuzzi implementation of PATE would provide the same level of classification performance with lower $\epsilon$ cost.

\subsection{Naïve Bayes}

We next implement a simple spam detection algorithm using the Spambase dataset from UCI Machine Learning Repository [Dheeru and Karra Taniskidou 2017]. The binary-labeled dataset (spam or non-spam) consists of 57 features, mostly of word frequencies for a set of given words, with additional features describing run lengths of certain sequences. We binarize all features from the data set to simplify the probability model described below (i.e., instead of how frequently a word appears on a scale of $[0,100]$, we only know whether the word was used (1) or not (0)). We can implement a more sophisticated Gaussian Nav̈e Bayes model that takes advantage of the frequency data using the same principles as in this experiment, but we chose to simplify the features to present a simpler model. We use 4500 samples for training and 100 samples for evaluation. Our privacy goal in this experiment is to limit an adversary's ability to guess whether a particular document was used to train the classification model.

A key assumption of the Naïve Bayes model is that given the class $y$ of a data point $\vec{x}$, all features are conditionally independent of each other. This assumption allows us to decompose the joint probability $P(\vec{x}, y)$ into the product $P(y) \cdot \Pi_{j} P\left(x_{j} \mid y\right)$, where $x_{j}$ represents the $j$-th coordinate of the binary vector $\vec{x}$. In our experimental setup, the $j$-th coordinate of a data point represents the presence of a word in the document. The goal of the Naïve Bayes model is to estimate the probabilities of $P(y=1)$ and $P\left(x_{j}=1 \mid y=1\right)$ and $P\left(x_{j}=1 \mid y=0\right)$ given the training data. Thus, when we get a new document $\vec{x}^{\prime}$, we can compare the probabilities

$$
P(y=1) \Pi_{j} P\left(x_{j}=x_{j}^{\prime} \mid y=1\right) \leq ? P(y=0) \Pi_{j} P\left(x_{j}=x_{j}^{\prime} \mid y=0\right)
$$

to make a prediction on whether the document is spam or not $(y=1$ or $y=0)$. Since we have 57 features, this gives us $57 \cdot 2+1=115$ parameters to estimate. 
Estimating the parameter $P(y=1)$ simply involves adding noise to the number of spam documents in the training set and dividing that count by the noised size of the training data set. We achieve this by first applying bmap to map each training data point to either 1 or 0 depending on its label, followed by bsum and Laplace mechanism to get a noised count. We can get a noised size of the training set through applying Laplace mechanism the training set's size.

Estimating the parameters $P\left(x_{j}=1 \mid y=1\right)$ and $P\left(x_{j}=1 \mid y=0\right)$ follows an essentially identical procedure. We first apply bmap to each training data point to map them to either 1 or 0 based on the values of $x_{j}$ and $y$, followed by a bsum operation to get the value of these conditional counts. We already computed the noised count of training samples with $y=1$ when estimating $P(y=1)$, but we perform the same procedure to compute a noised count of $y=0$. Dividing the noised conditional counts by the noised counts of $y=1$ and $y=0$ gives us the final estimates of the model parameters.

We achieve a training accuracy of 0.70 and test accuracy of 0.69 , with privacy costs $\epsilon=7.70$ and $\delta=0$. This classification accuracy is only slightly worse than the accuracy 0.72 of a non-private Naïve Bayes model that we implemented using binarized features from the same dataset.

\subsection{K-Means}

Finally, we perform a K-Means clustering experiment to evaluate Fuzzi's usability for an unsupervised learning task on the iris dataset from Fisher [1936]. This dataset contains three classes of iris flowers, with 50 flowers from each class. Each flower comes with four numeric features of petal and sepal dimensions and a label representing its class. Our experiment randomly selected one data point from each of the three classes as the initial public centroids; the Fuzzi program uses partition to map each data point to its closest centroid and create partitions accordingly. Other than the three data points used to initialize centroids, we used all other data for unsupervised training. (This experiment assumes a small part-in this case, three data points-of the training set is given as public information. Past work implementing differentially private K-Means made a similar assumption [Reed and Pierce 2010].)

On each pass over the training set, we first compute a noised sum of data points within each partition; we also compute noised sizes of each partition. We use these values to compute each partition's average point as the new centroids for the next pass. For evaluation, we classify all points within a partition with the majority label, and we obtain the accuracy of the clustering with these classifications. We do not use the labels for unsupervised training.

We found that the performance of the clustering algorithm varies depending on the initial centroids selected: running the experiment 100 times, all within 5 passes over the data set, we reach lowest accuracy of 0.55 and highest accuracy of 0.9 , with a median accuracy of 0.69 . Increasing the iteration count does not reduce this spread. We implemented a non-private version of the same algorithm, and achieved lowest accuracy 0.59, highest accuracy 0.96 and median accuracy of 0.59 on 100 experiments. Similar to Naïve Bayes, we see a slight drop in classification accuracy compared to the non-private implementation.

Each run has privacy cost $\epsilon=21.0$ and $\delta=0.0$. The large $\epsilon$ cost here is again related to the small size of the training set. In a small dataset, each data point has a larger impact on the released centroids; in order to reach a reasonable level of classification accuracy, we chose to apply the Laplace mechanism with a smaller noise level, resulting in larger $\epsilon$ cost.

\section{LIMITATIONS}

We briefly discuss some limitations and shortcomings of Fuzzi.

Limitation of sensitivity. Fuzzi's type system interface strikes a careful balance between expressiveness and complexity. Our approach is sufficient for expressing sensitivities of primitive values 
such as int and real and can capture a top-level sensitivity for vectors and bags; however, typing contexts cannot express sensitivities for individual values within a vector. For example, McSherry and Mironov developed a differentially private version of the recommender system based on Netflix user ratings [McSherry and Mironov 2009], where the sensitivity of inputs to the system is defined by the changes that may happen to a single row within a matrix, rather than the whole matrix. Fuzzi currently cannot carry out automatic inter-structure sensitivity derivation and cannot provide automatic differential privacy checking for McSherry and Mironov's algorithm.

Lack of support for abstraction. Vectors and bags are well studied objects in the differential privacy literature, and they have first class support in Fuzzi. However, Fuzzi does not provide facilities to specify general abstract data types and their neighboring relations. Fuzzi must know how to translate neighboring relation into an apRHL assertion, and this translation is not currently extensible. This limitation may force programmers to contort their code in order to represent a high-level concept through arrays. An example algorithm that cannot be adequately expressed in Fuzzi due to lack of abstraction is the binary mechanism [Chan et al. 2011], which builds a tree of partial sums of the input data and accumulates a statistic whose sensitivity is proportional to the depth of the tree.

Potential Vulnerabilities. Fuzzi's semantics uses real numbers as a model for the type real. However, the implementation uses floating point numbers. As shown by Mironov [2012], using the Laplace mechanism in this setting may result in vulnerable distributions that can compromise the original sensitive data. Although Fuzzi guarantees co-termination over neighboring data, it is vulnerable to timing channel attacks [Haeberlen et al. 2011]. A Fuzzi program that uses sensitive loop conditions may result in vastly different execution duration. This side channel allows an attacker to distinguish runs with high confidence. The first issue can be alleviated by a careful implementation of Laplace mechanism that incorporates Mironov's mitigation strategy, while the second issue is more fundamental-Fuzzi's type system needs to approximately measure the execution time, which we did not address in this work.

Performance concern due to copy assignments. Fuzzi uses copy assignments for arrays. We have worked with relatively small datasets in the experiments, and the sizes of these arrays have not caused severe performance problems in our experiments. However, today's machine learning tasks typically operate on datasets that are many orders of magnitude larger, and Fuzzi likely cannot handle computations over these datasets efficiently. To adapt Fuzzi's theory for a semantics that allows sharing, we need to create a new flavor of apRHL that can reason about heaps. One potential direction is to integrate separation logic [Reynolds 2002] into apRHL.

\section{RELATED WORK}

Query languages. McSherry introduced Privacy Integrade Queries (PINQ) as an embedded query language extension for the C\# programming language [McSherry 2009]. PINQ pioneered languagelevel support for differential privacy by analyzing sensitivities for SQL-like queries and releasing noised results of these queries using the Laplace mechanism. Fuzzi's partition extension takes inspiration from PINQ's partition operator, adapting it to an imperative program that computes over arrays.

The FLEX framework [Johnson et al. 2018] allows programmers to run differentially private SQL queries against a private database. FLEX uses an elastic sensitivity technique to support SQL queries with joins based on equality. Fuzzi focuses on adding support of Differential Privacy to a general-purpose imperative language; however, the theory around elastic sensitivities could inspire future extensions to the Fuzzi type system. 
DJoin [Narayan and Haeberlen 2012] runs SQL queries over databases distributed over many machines. The distributed nature of the data is not just a question of size, but may be due to the fact that different databases may be owned by different organizations that do not wich to share them; there simply is no single way to get all the data in the same place. (For example, analysts may want to correlate travel data with illness diagnosis data, with the former provided by airline companies while the latter provided by hospitals.) Fuzzi does not address distributed computations: it runs on a single machine and assumes data is already in the memory of this machine.

Fuzz and related languages. Fuzz is a higher-order functional programming language with a sensitivity-tracking type system and differentially private primitives [Reed and Pierce 2010]. Fuzzi's sensitivity type system is inspired by Fuzz, but differs in that Fuzzi separately tracks the sensitivity of each value in the store (which may be change as the program assigns to variables), while Fuzz tracks only function sensitivity. Also, Fuzz's type system is restricted to $(\epsilon, 0)$-differential privacy, while Fuzzi generalizes this to $(\epsilon, \delta)$-differential privacy.

DFuzz [Gaboardi et al. 2013] extends Fuzz with linear indexed types and dependent types, allowing programmers to abstract types over sensitivity annotations. Compared to DFuzz, both Fuzz and Fuzzi only allow purely numeric values as sensitivity annotations in types. This additional level of expressiveness admits programs whose sensitivities and privacy costs scale with input sensitivities. Although Fuzzi does not allow such indexed types, the extension mechanism does allow language developers to add typing rules quantified over unknown constants (such as the loop count in ADV-Comp for advanced composition); this provides another way for programmers to write programs whose privacy costs scale with program constants.

AdaptiveFuzz [Winograd-Cort et al. 2017] extends Fuzz by using staged computation and stream semantics to implement a powerful composition mechanism called Privacy Filters [Rogers et al. 2016]. These give programmers the freedom to run future computations based on results released from earlier differentially private computations. This allows, for example, programmers to stop a private gradient descent loop as soon as accuracy reaches a desired threshold, rather than fixing the number of iterations ahead of time. Fuzzi implements advanced composition for improved privacy cost aggregation, but privacy filters are not yet formalized in either apRHL or Fuzzi.

Duet [Near et al. 2019] is a higher-order functional language that provides $(\epsilon, \delta)$-differential privacy. Fuzz's original type system relies on composition properties that break down when generalized to cases where $\delta>0$. As an example, an $(\epsilon, 0)$-DP Fuzz function $f$ that takes a 1sensitive dataset as input has the property that, when $f$ runs on a 2-sensitive dataset, the privacy costs scales accordingly to $(2 \epsilon, 0)$-DP. However, if $f$ is a general $(\epsilon, \delta)$-DP computation on 1-sensitive datasets, it is not true that running $f$ on a 2 -sensitive dataset is $(2 \epsilon, 2 \delta)$-DP. Duet solves this problem by separating its type system into two disjoint parts: one that keeps track of sensitivities and allows scaling and another that keeps track of privacy costs and disallows scaling. In Fuzzi, we have a similar separation: the typing contexts of a command sequence are strict pre-conditions and post-conditions and do not allow scaling, except for commands typechecked with the linear typing judgements that explicitly allow scaling of sensitivities in the pre- and post-condition. These linear commands are deterministic and cannot use the Laplace mechanism by definition, so scaling their typing judgements' sensitivities does not bring up the same issues that Fuzz has.

Verification systems. Albarghouthi and Hsu [2017] developed an automated differential privacy proof synthesis system based on the idea of coupling. Fuzzi and apRHL use the same mathematical device to simplify relational reasoning between non-deterministic outputs from the Laplace mechanism.

LightDP is an imperative language with semi-automatic typechecking that uses dependent types to prove differential privacy properties of a program [Zhang and Kifer 2017]. LightDP's type system 
also keeps track of distances of variables between executions on neighboring inputs. A major difference from Fuzzi is that LightDP's type system tracks the exact distance between variables through a dependent type system, while Fuzzi tracks upper bounds on the distance between variables. LightDP elaborates the source code into a slightly extended language that explicitly keeps track of privacy costs in a distinguished variable and then uses a MaxSMT solver to discharge the generated verification conditions in the process of typechecking. Fuzzi typing rules' soundness are proven ahead of time, and Fuzzi's sensitivity checking process does not generate new proof obligations. Due to this design, Fuzzi does not require a constraint solver to aid in typechecking.

The EasyCrypt toolset allows developers to construct machine-checkable proofs of relational properties for probabilistic computations [EasyCrypt Development Team 2018]. EasyCrypt has a development branch that focuses on Differential Privacy verification through apRHL. EasyCrypt provides built-in support of apRHL proof rules, and also supports termination analysis through a compatible program logic pHL (Probabilistic Hoare Logic). Fuzzi’s development does not connect with EasyCrypt's apRHL implementation, but this is a potential future direction for rigorously checking Fuzzi's theories.

Testing Differential Privacy. Ding et al. [2018] developed a statistical testing framework for detecting violations of differential privacy of Python programs. This framework performs static analysis on Python code and generates inputs that seem likely to violate differential privacy based on this analysis. It also repeatedly executes the program to collect statistical evidence of violations of differential privacy. This framework demonstrates the potential for a lighter-weight approach to providing differential privacy guarantees; we could potentially apply the same methodology to aid Fuzzi extension designers by testing typing rules before formally proving their soundness.

High-level frameworks. The PSI private data sharing interface [Gaboardi et al. 2016] is designed to enable non-expert users and researchers to both safely deposit private data and apply a select set of differentially private algorithms to collect statistics from the deposited data. Fuzzi, on the other hand, is designed only for the task of implementing differentially private algorithms. It expects its users to have some familiarity with key concepts such as sensitivity and privacy budget, and it allows power users to extend its typechecker for more sophisticated programs.

The $\epsilon$ KTELLO Framework [Zhang et al. 2018] provides a set of expressive high-level combinators for composing algorithms, with the guarantee that any algorithm composed of $\epsilon$ KTELLO combinators automatically satisfies differential privacy. The $\epsilon$ KTELLO framework allows users to customize differentially private algorithms in order to achieve higher utility from querying private data. Fuzzi, by contrast, is an attempt at building a rather low-level core language with support for Differential Privacy. A future direction could be to build a high-level framework like $\epsilon$ KTELLO over Fuzzi, providing both expressive combinators and automatic verification of Differential Privacy for the implementation of these combinators in the same system.

\section{CONCLUSION AND FUTURE WORK}

The rise of Differential Privacy calls for reliable, yet familiar tools that help programmers control privacy risks. Fuzzi gives programmers a standard imperative language with automated privacy checking, which can be enriched by expert users with extensions whose privacy properties are proved in a special-purpose relational logic.

Many avenues for improvement still remain. (1) We can enrich the set of Fuzzi extensions to further increase Fuzzi's utility. For example, adding Report Noisy Max would allow an analyst to find the largest value in a vector with small privacy cost; the Exponential mechanism would allow programs to release categorical data (as opposed to numerical) with differential privacy guarantees [Dwork and Roth 2014]. We expect both mechanisms can be formalized in apRHL [Barthe 
et al. 2016] and added to Fuzzi. (2) We can engineer typechecker plugins that dynamically load new extension typing rules to make Fuzzi's implementation more flexible. (At the moment, adding an extension typing rule requires editing the typechecker sources.) (3) We can formalize Privacy Filters [Rogers et al. 2016] in apRHL and add adaptive composition to Fuzzi. This would allow programmers to use the adaptive aggregation mechanism from AdaptiveFuzz in Fuzzi. (4) We can implement Fuzzi as a formalized framework in Coq [Coq Development Team 2018]. This would allow power users to write machine-checked proofs of extension typing rules. (5) We can incorporate proof synthesis techniques to automatically search for privacy proofs for extensions, following Albarghouthi and Hsu [2017], who demonstrated the effectiveness of synthesizing privacy proofs for interesting differential privacy mechanisms. Proof synthesis could streamline prototyping new Fuzzi extensions and their typing rules.

\section{ACKNOWLEDGMENTS}

We are grateful to Justin Hsu, David Darais, and Penn PLClub for their comments, and we thank the anonymous ICFP reviewers for their detailed and helpful feedback. This work was supported in part by the National Science Foundation under grants CNS-1065060 and CNS-1513694.

\section{REFERENCES}

Martin Abadi, Andy Chu, Ian Goodfellow, H. Brendan McMahan, Ilya Mironov, Kunal Talwar, and Li Zhang. 2016. Deep Learning with Differential Privacy. In Proceedings of the 2016 ACM SIGSAC Conference on Computer and Communications Security (CCS '16). ACM, New York, NY, USA, 308-318. https://doi.org/10.1145/2976749.2978318

Amal Ahmed. 2006. Step-Indexed Syntactic Logical Relations for Recursive and Quantified Types. In Programming Languages and Systems, Peter Sestoft (Ed.). Springer Berlin Heidelberg, Berlin, Heidelberg, 69-83.

Aws Albarghouthi and Justin Hsu. 2017. Synthesizing Coupling Proofs of Differential Privacy. Proc. ACM Program. Lang. 2, POPL, Article 58 (Dec. 2017), 30 pages. https://doi.org/10.1145/3158146

Andrew W. Appel and David McAllester. 2001. An Indexed Model of Recursive Types for Foundational Proof-carrying Code. ACM Trans. Program. Lang. Syst. 23, 5 (Sept. 2001), 657-683. https://doi.org/10.1145/504709.504712

Andrew W. Appel, Paul-André Melliès, Christopher D. Richards, and Jérôme Vouillon. 2007. A Very Modal Model of a Modern, Major, General Type System. In Proceedings of the 34th Annual ACM SIGPLAN-SIGACT Symposium on Principles of Programming Languages (POPL '07). ACM, New York, NY, USA, 109-122. https://doi.org/10.1145/1190216.1190235

Apple. 2017. Apple Differential Privacy Whitepaper. https://images.apple.com/privacy/docs/Differential_Privacy_Overview. pdf

Patrick Bahr and Tom Hvitved. 2011. Compositional Data Types. In Proceedings of the Seventh ACM SIGPLAN Workshop on Generic Programming (WGP '11). ACM, New York, NY, USA, 83-94. https://doi.org/10.1145/2036918.2036930

Gilles Barthe, Marco Gaboardi, Benjamin Grégoire, Justin Hsu, and Pierre-Yves Strub. 2016. Proving Differential Privacy via Probabilistic Couplings. In Proceedings of the 31st Annual ACM/IEEE Symposium on Logic in Computer Science (LICS '16). ACM, New York, NY, USA, 749-758. https://doi.org/10.1145/2933575.2934554

Nick Benton. 2004. Simple Relational Correctness Proofs for Static Analyses and Program Transformations. In Proceedings of the 31st ACM SIGPLAN-SIGACT Symposium on Principles of Programming Languages (POPL '04). ACM, New York, NY, USA, 14-25. https://doi.org/10.1145/964001.964003

T.-H. Hubert Chan, Elaine Shi, and Dawn Song. 2011. Private and Continual Release of Statistics. ACM Trans. Inf. Syst. Secur. 14, 3, Article 26 (Nov. 2011), 24 pages. https://doi.org/10.1145/2043621.2043626

Yan Chen and Ashwin Machanavajjhala. 2015. On the Privacy Properties of Variants on the Sparse Vector Technique. CoRR abs/1508.07306 (2015). arXiv:1508.07306 http://arxiv.org/abs/1508.07306

The Coq Development Team. 2018. The Coq Proof Assistant Reference Manual, version 8.8. http://coq.inria.fr

Dua Dheeru and Efi Karra Taniskidou. 2017. UCI Machine Learning Repository. http://archive.ics.uci.edu/ml

Zeyu Ding, Yuxin Wang, Guanhong Wang, Danfeng Zhang, and Daniel Kifer. 2018. Detecting Violations of Differential Privacy. In Proceedings of the 2018 ACM SIGSAC Conference on Computer and Communications Security (CCS '18). ACM, New York, NY, USA, 475-489. https://doi.org/10.1145/3243734.3243818

Cynthia Dwork, Frank McSherry, Kobbi Nissim, and Adam Smith. 2006. Calibrating Noise to Sensitivity in Private Data Analysis. In Proceedings of the Third Conference on Theory of Cryptography (TCC'06). Springer-Verlag, Berlin, Heidelberg, 265-284. https://doi.org/10.1007/11681878_14

Cynthia Dwork and Aaron Roth. 2014. The Algorithmic Foundations of Differential Privacy. Found. Trends Theor. Comput. Sci. 9, 3\&\#8211;4 (Aug. 2014), 211-407. https://doi.org/10.1561/0400000042 
Cynthia Dwork, Guy Rothblum, and Salil Vadhan. 2010. Boosting and Differential Privacy. In Proceedings of the 51st Annual IEEE Symposium on Foundations of Computer Science (FOCS '10). IEEE, IEEE, Las Vegas, NV, 51-60. http: //dx.doi.org/10.1109/FOCS.2010.12

The EasyCrypt Development Team. 2018. EasyCrypt Reference Manual, version 1.x. https://www.easycrypt.info/ documentation/refman.pdf

Úlfar Erlingsson, Vasyl Pihur, and Aleksandra Korolova. 2014. RAPPOR: Randomized Aggregatable Privacy-Preserving Ordinal Response. In Proceedings of the 2014 ACM SIGSAC Conference on Computer and Communications Security (CCS '14). ACM, New York, NY, USA, 1054-1067. https://doi.org/10.1145/2660267.2660348

R. A. Fisher. 1936. The Use of Multiple Measurements in Taxonomic Problems. Annals of Eugenics 7, 7 (1936), 179-188.

Dan Frumin, Robbert Krebbers, and Lars Birkedal. 2018. ReLoC: A Mechanised Relational Logic for Fine-Grained Concurrency. In Proceedings of the 33rd Annual ACM/IEEE Symposium on Logic in Computer Science (LICS '18). ACM, New York, NY, USA, 442-451. https://doi.org/10.1145/3209108.3209174

Marco Gaboardi, Andreas Haeberlen, Justin Hsu, Arjun Narayan, and Benjamin C. Pierce. 2013. Linear Dependent Types for Differential Privacy. In Proceedings of the 40th Annual ACM SIGPLAN-SIGACT Symposium on Principles of Programming Languages (POPL '13). ACM, New York, NY, USA, 357-370. https://doi.org/10.1145/2429069.2429113

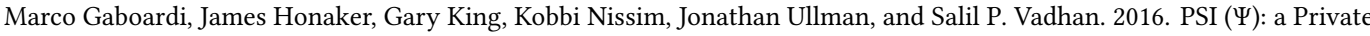
data Sharing Interface. CoRR abs/1609.04340 (2016). arXiv:1609.04340 http://arxiv.org/abs/1609.04340

Andreas Haeberlen, Benjamin C. Pierce, and Arjun Narayan. 2011. Differential Privacy Under Fire. In Proceedings of the 20th USENIX Conference on Security (SEC'11). USENIX Association, Berkeley, CA, USA, 33-33. http://dl.acm.org/citation.cfm? $\mathrm{id}=2028067.2028100$

C. A. R. Hoare. 1969. An Axiomatic Basis for Computer Programming. Commun. ACM 12, 10 (Oct. 1969), 576-580 https://doi.org/10.1145/363235.363259

Justin Hsu. 2018. Private Communication.

Noah Johnson, Joseph P. Near, and Dawn Song. 2018. Towards Practical Differential Privacy for SQL Queries. Proc. VLDB Endow. 11, 5 (Jan. 2018), 526-539. https://doi.org/10.1145/3187009.3177733

Ralf Jung, Jacques-Henri Jourdan, Robbert Krebbers, and Derek Dreyer. 2017. RustBelt: Securing the Foundations of the Rust Programming Language. Proc. ACM Program. Lang. 2, POPL, Article 66 (Dec. 2017), 34 pages. https://doi.org/10. 1145/3158154

Y. Lecun, L. Bottou, Y. Bengio, and P. Haffner. 1998. Gradient-based learning applied to document recognition. Proc. IEEE 86, 11 (Nov 1998), 2278-2324. https://doi.org/10.1109/5.726791

Yann LeCun and Corinna Cortes. 2010. MNIST handwritten digit database. http://yann.lecun.com/exdb/mnist/. (2010). http://yann.lecun.com/exdb/mnist/

Min Lyu, Dong Su, and Ninghui Li. 2016. Understanding the Sparse Vector Technique for Differential Privacy. CoRR abs/1603.01699 (2016). arXiv:1603.01699 http://arxiv.org/abs/1603.01699

H. Brendan McMahan, Daniel Ramage, Kunal Talwar, and Li Zhang. 2018. Learning Differentially Private Recurrent Language Models. In International Conference on Learning Representations. https://openreview.net/forum?id=BJ0hF1Z0b

Frank McSherry and Ilya Mironov. 2009. Differentially Private Recommender Systems: Building Privacy into the Netflix Prize Contenders. In Proceedings of the 15th ACM SIGKDD International Conference on Knowledge Discovery and Data Mining (KDD '09). ACM, New York, NY, USA, 627-636. https://doi.org/10.1145/1557019.1557090

Frank D. McSherry. 2009. Privacy Integrated Queries: An Extensible Platform for Privacy-preserving Data Analysis. In Proceedings of the 2009 ACM SIGMOD International Conference on Management of Data (SIGMOD '09). ACM, New York, NY, USA, 19-30. https://doi.org/10.1145/1559845.1559850

Microsoft. 2017. Collecting telemetry data privately. https://www.microsoft.com/en-us/research/blog/collecting-telemetrydata-privately/

Ilya Mironov. 2012. On Significance of the Least Significant Bits for Differential Privacy. In Proceedings of the 2012 ACM Conference on Computer and Communications Security (CCS '12). ACM, New York, NY, USA, 650-661. https: //doi.org/10.1145/2382196.2382264

Aref N. Dajani, Amy D. Lauger, Phyllis E. Singer, Daniel Kifer, Jerome P. Reiter, Ashwin Machanavajjhala, Simson L. Garfinkel, Scot A. Dahl, Matthew Graham, Vishesh Karwa, Hang Kim, Philip Leclerc, Ian M. Schmutte, William N. Sexton, Lars Villhuber, and John M. Abowd. 2017. The modernization of statistical disclosure limitation at the U.S. Census Bureau. (September 2017). https://www2.census.gov/cac/sac/meetings/2017-09/statistical-disclosure-limitation.pdf [Online; posted September-2017].

Arjun Narayan and Andreas Haeberlen. 2012. DJoin: Differentially Private Join Queries over Distributed Databases. In Proceedings of the 10th USENIX Conference on Operating Systems Design and Implementation (OSDI'12). USENIX Association, Berkeley, CA, USA, 149-162. http://dl.acm.org/citation.cfm?id=2387880.2387895

Joseph P. Near, David Darais, Tim Stevens, Paranav Gaddamadugu, Lun Wang, Neel Somani, Mu Zhang, Nikhil Sharma, Alex Shan, and Dawn Song. 2019. (2019). http://david.darais.com/assets/papers/duet/duet.pdf 
Travis E. Oliphant. 2015. Guide to NumPy (2nd ed.). CreateSpace Independent Publishing Platform, USA.

Nicolas Papernot, Martín Abadi, Úlfar Erlingsson, Ian Goodfellow, and Kunal Talwar. 2016. Semi-supervised Knowledge Transfer for Deep Learning from Private Training Data. arXiv:1610.05755 [cs, stat] (Oct. 2016). http://arxiv.org/abs/1610. 05755 arXiv: 1610.05755 .

Jason Reed and Benjamin C. Pierce. 2010. Distance Makes the Types Grow Stronger: A Calculus for Differential Privacy. SIGPLAN Not. 45, 9 (Sept. 2010), 157-168. https://doi.org/10.1145/1932681.1863568

John C. Reynolds. 2002. Separation Logic: A Logic for Shared Mutable Data Structures. In Proceedings of the 17th Annual IEEE Symposium on Logic in Computer Science (LICS '02). IEEE Computer Society, Washington, DC, USA, 55-74. http: //dl.acm.org/citation.cfm?id=645683.664578

Ryan M Rogers, Aaron Roth, Jonathan Ullman, and Salil Vadhan. 2016. Privacy Odometers and Filters: Pay-as-you-Go Composition. In Advances in Neural Information Processing Systems 29, D. D. Lee, M. Sugiyama, U. V. Luxburg, I. Guyon, and R. Garnett (Eds.). Curran Associates, Inc., 1921-1929. http://papers.nips.cc/paper/6170-privacy-odometers-andfilters-pay-as-you-go-composition.pdf

Tetsuya Sato. 2016. Approximate Relational Hoare Logic for Continuous Random Samplings. Electronic Notes in Theoretical Computer Science 325 (2016), 277 - 298. https://doi.org/10.1016/j.entcs.2016.09.043 The Thirty-second Conference on the Mathematical Foundations of Programming Semantics (MFPS XXXII).

Tetsuya Sato, Gilles Barthe, Marco Gaboardi, Justin Hsu, and Shin-ya Katsumata. 2019. Approximate Span Liftings. CoRR abs/1710.09010 (2019). arXiv:1710.09010 http://arxiv.org/abs/1710.09010

Wouter Swierstra. 2008. Data Types à La Carte. F. Funct. Program. 18, 4 (July 2008), 423-436. https://doi.org/10.1017/ S0956796808006758

Daniel Winograd-Cort, Andreas Haeberlen, Aaron Roth, and Benjamin C. Pierce. 2017. A Framework for Adaptive Differential Privacy. Proc. ACM Program. Lang. 1, ICFP, Article 10 (Aug. 2017), 29 pages. https://doi.org/10.1145/3110254

Danfeng Zhang and Daniel Kifer. 2017. LightDP: Towards Automating Differential Privacy Proofs. SIGPLAN Not. 52,1 (Jan. 2017), 888-901. https://doi.org/10.1145/3093333.3009884

Dan Zhang, Ryan McKenna, Ios Kotsogiannis, Michael Hay, Ashwin Machanavajjhala, and Gerome Miklau. 2018. Ektelo: A Framework for Defining Differentially-Private Computations. In SIGMOD Conference. 